\section{OPEN ACCESS}

Edited by:

Robert Ernst,

Saarland University, Germany

Reviewed by:

Erdinc Sezgin,

Karolinska Institutet (KI), Sweden

Edward Lyman,

University of Delaware, United States

*Correspondence:

Vahid Sandoghdar

vahid.sandoghdar@mpl.mpg.de

tThese authors have contributed equally to this work

Specialty section:

This article was submitted to Membrane Traffic

a section of the journal

Frontiers in Cell and Developmental

Biology

Received: 31 July 2020

Accepted: 05 October 2020

Published: 03 November 2020

Citation:

Taylor RW, Holler C

Mahmoodabadi RG, Küppers $M$,

Dastjerdi HM, Zaburdaev V,

Schambony A and Sandoghdar V

(2020) High-Precision

Protein-Tracking With Interferometric

Scattering Microscopy.

Front. Cell Dev. Biol. 8:590158.

doi: 10.3389/fcell.2020.590158

\title{
High-Precision Protein-Tracking With Interferometric Scattering Microscopy
}

\begin{abstract}
Richard W. Taylor ${ }^{1,2+}$, Cornelia Holler ${ }^{1,2 \dagger}$, Reza Gholami Mahmoodabadi ${ }^{1,2}$, Michelle Küppers ${ }^{1,2,3}$, Houman Mirzaalian Dastjerdi 1,2,4, Vasily Zaburdaev ${ }^{2,5}$, Alexandra Schambony ${ }^{1,2,5}$ and Vahid Sandoghdar ${ }^{1,2,3 *}$
\end{abstract}

${ }^{1}$ Max Planck Institute for the Science of Light, Erlangen, Germany, ${ }^{2}$ Max-Planck-Zentrum für Physik und Medizin, Erlangen, Germany, ${ }^{3}$ Department of Physics, Friedrich Alexander University Erlangen-Nuremberg, Erlangen, Germany, ${ }^{4}$ Department of Computer Science, Friedrich Alexander University Erlangen-Nuremberg, Erlangen, Germany, ${ }^{5}$ Department of Biology, Friedrich Alexander University Erlangen-Nuremberg, Erlangen, Germany

The mobility of proteins and lipids within the cell, sculpted oftentimes by the organization of the membrane, reveals a great wealth of information on the function and interaction of these molecules as well as the membrane itself. Single particle tracking has proven to be a vital tool to study the mobility of individual molecules and unravel details of their behavior. Interferometric scattering (iSCAT) microscopy is an emerging technique well-suited for visualizing the diffusion of gold nanoparticle-labeled membrane proteins to a spatial and temporal resolution beyond the means of traditional fluorescent labels. We discuss the applicability of interferometric single particle tracking (iSPT) microscopy to investigate the minutia in the motion of a protein through measurements visualizing the mobility of the epidermal growth factor receptor in various biological scenarios on the live cell.

Keywords: interferometric scattering microscopy, iSCAT, iSPT, single particle tracking, live cell imaging, membrane organization, epidermal growth factor receptor

\section{INTRODUCTION}

All cells are enclosed by an outer plasma membrane and, in addition, eukaryotic cells are commonly compartmentalized by internal membranes into cell organelles, to generate specialized functional entities. The plasma membrane acts as a barrier, transport, and communication platform between the cell and its environment that separates the interior of the cell from its surroundings, controls the flux of ions and nutrients and mediates sending and sensing of signals to and from the cell. These multiple functions of the membrane are enabled by its complex composition of phospholipids, sphingo- and glycolipids, cholesterol, and proteins.

The plasma membrane is also a carefully regulated and highly dynamic structure which sculpts the mobility of the proteins and lipids of which it is composed (Krapf, 2018; Jacobson et al., 2019). Our view on the organizational motifs of the plasma membrane has expanded greatly over the last few decades following much investigation and advances in single-particle imaging microscopies. Present wisdom informs that the plasma membrane is organized over many length scales (Destainville et al., 2008; Krapf, 2018; Jacobson et al., 2019), with motifs including cellular domains such as cell-cell contact sites or the apical surface of epithelial cells being strictly separated from the basolateral surface of the membrane by cell-cell adhesion molecules. In addition, other 
motifs include cytoskeletal actin-mesh induced microscale compartmentalization (Fujiwara et al., 2002, 2016; Jaqaman and Grinstein, 2012), nanoscale transient raft domains (Pike, 2006; Jacobson et al., 2007; Sezgin et al., 2017), and molecular-level crowding from protein-protein interactions (Jin and Verkman, 2007; Dix and Verkman, 2008).

These organizational motifs provide a mechanism for spatial and temporal control of the lipids and proteins by hierarchically restricting their freedom of movement. Doing so allows for regions of specific composition and hence dedicated function which is required for a diverse array of physiological processes such as signal transduction, directed transport across a membrane or cell-cell-communication (Destainville et al., 2008; Krapf, 2018). In signaling, one requires the formation of nanoscale signaling domains which comprise selected proteins. By transiently confining respective proteins, these domains are believed to regulate signaling events by changing the local concentration of proteins and as a consequence modulating efficient protein-protein interactions. In contrast, they also have the capacity to prevent signaling altogether in the absence of a stimulus through exclusionary segregation of the membrane components (Krapf, 2018).

The ability to track the motion of proteins within the membrane on the one hand provides insights on the paths a particular protein takes during the period of observation and on the other indirectly reveals obstacles, boundaries, or accelerators that affect its mobility. One thus stands to gain further insight into the functionality and structure of the membrane by observing the lateral mobility of membrane proteins as these molecules serve as ideal probes for this complex environment. Unsurprisingly, tracking protein mobility has been an important target of research over the last decades, with numerous optical techniques having been developed to meet this challenge (Jaqaman and Grinstein, 2012). Studies at the single molecule level have shown to be extremely fruitful since they allow for direct visualization of the behavior of individual molecules and their molecular interactions, no matter how rare or infrequent, without fear of being lost to an ensemble observation.

Single particle tracking (SPT) of protein and lipid mobility has proven to be an exceptionally valuable and productive technique in this effort to decipher the interactions that constitute the organizing principles of the membrane (Alcor et al., 2009; Kusumi et al., 2014; Manzo and Garcia-Parajo, 2015; Shen et al., 2017; Ye et al., 2019). SPT microscopy is typically accomplished by localizing the position of the membrane molecule through attachment of an optical label, be the label a fluorescent molecule (Wieser and Schütz, 2008; Albrecht et al., 2015), quantum dot (Pinaud et al., 2010; Clausen and Lagerholm, 2013), or nanoparticle (Kusumi et al., 1993; Fujiwara et al., 2002). The purpose is then to reveal the path taken in the membrane by the molecule as detected at various temporal resolutions depending on the method of choice. While low temporal resolution can provide only partial information about the motion of the molecule without revealing the fine details of its diffusion, imaging with high frame rates effectively visualizes the molecular trajectories resultant from fast and short-lived interactions within the membrane.
Fluorescence-based labels have been enormously popular for single-molecule imaging since they enable precise distinction between signal and background during imaging. However, fluorescence imposes several important limitations which restrict how well one can track the mobility of a membrane molecule. Firstly, the finite rate by which light is emitted from the fluorophore limits how well the position of the labeled protein can be determined. For a molecule to emit a fluorescence photon, an electron within the molecule must become promoted into a higher energy level through absorption of a photon. On relaxation of the electron, a fluorescent photon is emitted, with the whole process taking several nanoseconds. The rate at which the label can emit photons is a critical quantity in SPT as the measured number of photons determines directly the precision to which the location of an emitter can be determined (Thompson et al., 2002; Deschout et al., 2014). Since the emission rate is depending on the excitation lifetime, one therefore finds that for nanosecond lifetimes the best attainable resolutions are on the order of tens of nanometers per millisecond of recording.

In addition, fluorescent dyes are vulnerable to several processes that result in the emission of no light which is naturally detrimental for tracking microscopies. Firstly, photochemical processes can occur which alter the wavelength needed for the emitter to become excited or to fluoresce, known as photobleaching. Secondly, it is possible for the electron within the fluorophore to become temporarily trapped in a transient state that is dark to the illumination wavelength, a process commonly referred to as blinking. Owing to these issues, there have been many efforts to investigate mechanisms for label-free imaging of bio-membranes (Mashaghi et al., 2014).

The desire to gain an ever greater insight into the precise mobility of single molecules within the membrane has motivated the search for alternative optical labels that can offer improved spatial and temporal resolutions in SPT microscopy. Scattering is an attractive alternative because it does not encounter the critical limitations previously described for fluorophores. Furthermore, the permanence of scattering also enables for the scattering label to be tracked for an essentially endless period, limited only by the sample itself, and so opening the possibility to watch entire biological processes in a complete uninterrupted fashion.

The first experiments that showed the potential for scattering labels were pioneered as far back as the late 1980s (De Brabander et al., 1986, 1988; Geerts et al., 1987). These efforts laid the foundation for the seminal investigations by Kusumi et al. whose high-speed single particle measurements revealed the existence of diffusion barriers within the plasma membrane and founded the concept of compartmentalized organization of the membrane itself (Kusumi et al., 1993, 2005; Fujiwara et al., 2002).

The challenge faced in performing SPT microscopy with nanoparticle labels is that the scattering signal is comparatively small and accompanied by a large imaging background from the remaining sample which must be tackled. Interferometric scattering (iSCAT) microscopy is an emerging technique which confronts these issues directly (Lindfors et al., 2004; Taylor and Sandoghdar, 2019; Taylor et al., 2019; Young and Kukura, 2019). With iSCAT, it recently has been shown to be possible to track the 
diffusion of labeled proteins on the membrane of artificial and live cells (Spindler et al., 2016; Huang et al., 2017a; de Wit et al., 2018; Taylor et al., 2019; Mazaheri et al., 2020) with nanometric precision in all three-dimensions with microsecond temporal resolution (Taylor et al., 2019; Mahmoodabadi et al., 2020). The recent advances of interferometric SPT (iSPT) is further spurring new efforts to understand and model the plasma membrane (Lyman et al., 2018).

In this work, we show how iSCAT microscopy is highly suited for investigating the mobility of a transmembrane signaling protein to fine detail within the live cell membrane. We chose the epidermal growth factor receptor (EGFR) as a candidate since EGFR is a prototypical receptor tyrosine kinase belonging to the ErbB family and is a key regulator of cell proliferation, growth, survival, and differentiation in mammalian cells (Oda et al., 2005). EGFR is an 1,186 amino acid single-pass transmembrane glycoprotein that binds and is activated by ligands of the Epidermal Growth Factor (EGF)-family with 11 known members in humans (Roskoski, 2014).

In its inactive state, EGFR is mostly residing and traveling within the plasma membrane either as a monomer or as preformed but inactive dimers, with the ability to continuously switch between these two states (Chung et al., 2010). To be primed for signaling, however, EGFR must be in the dimerised state in order to become active after EGF ligand binding. Signaling is initiated by autophosphorylation of the intracellular part of the receptor, which constitutes binding interfaces for signaling proteins from which the signal is further transduced along the signaling cascade. Once activated, EGFR becomes rapidly endocytosed and continues signaling in endocytic compartments until the receptors either are returning to the inactive state and recycled back to the membrane or become degraded via the lysosomal pathway (Linggi and Carpenter, 2006; Sorkin and Goh, 2009; Arteaga and Engelman, 2014; Roskoski, 2014; Tomas et al., 2014; Kovacs et al., 2015; Freed et al., 2017).

Since EGFR signaling strongly depends on receptor mobility as outlined above and aberrant signaling of EGFR is associated with various cancers by promoting oncogenic signaling, this receptor has been widely investigated as a model system to explore how the nanoscale organization of the plasma membrane affects signaling function (Oda et al., 2005; Erazo-Oliveras et al., 2018). For this reason, EGFR has also served as a model membrane protein for numerous SPT microscopies (Kusumi et al., 1993; Sako et al., 2000; Abulrob et al., 2010; Chung et al., 2010; Bag et al., 2015; Liu et al., 2016).

Here, we show how the high spatial and temporal resolution of iSPT can be used to uncover fine details in the trajectories of an EGFR in scenarios such as diffusion in the membrane as well as applicability in investigating processes such as endocytosis and trafficking. We discuss several methods for statistical quantification of single particle trajectories that take advantage of the high temporal resolution to reveal detailed information about protein mobility in the membrane such as nanoscale sub-diffusion and confinement events. We first begin by introducing the principle of iSPT microscopy and in particular how three-dimensional (3D) nanometric particle localization is accomplished.

\section{2. iSPT ON THE LIVE CELL}

\subsection{SPT Microscopy: Interferometric Scattering vs. Fluorescence}

In fluorescence-based SPT, one follows the molecule of interest by detecting the light emitted by an attached fluorescent label. Since the light emitted by the label is shifted to a longer wavelength than that used for excitation, one can exclusively detect the labeled protein through the use of convenient wavelengthselective filters. This detection strategy has proven enormously successful, with single-molecule sensitivity now being something widely accessible to many laboratories.

However, the aforementioned inconsistent and low number of photons emitted through fluorescence curtails the resolution and fidelity by which one may identify the position of the target molecule. This problem is further compounded with the presence of detection noise which is often of a similar intensity to the weak probe signal.

Light scattering is an alternative optical process to fluorescence which overcomes many of these difficulties. In light scattering, the incoming light briefly couples to the electrons in the material before being swiftly re-radiated away. For this reason, whilst not all materials can fluoresce, all are able to scatter light. Moreover, light scattering is not vulnerable to the processes of blinking and bleaching as encountered with fluorescence.

One advantage of light scattering is that the interaction between the photon and the electron is near-instantaneous. For a metal nanoparticle, such as gold, this interaction can be as fast as $10 \mathrm{fs}$ and hence one can generate a million scattered photons in the same time it would take to generate a single fluorescence photon.

The process of light scattering also has the crucial property of being coherent, a feature not shared by fluorescence. In being coherent, the light scattered by the particle retains the same temporal signature as the illuminating light, meaning they share the same phase difference throughout time. Hence, two light beams can be meaningfully added together in an optical process known as interference. The advantage in interfering two optical beams together is that if one of the beams is weak in amplitudefor example as occurs with nanoscale scattering, then one can boost the effective amplitude of this weak signal by mixing with a stronger coherent companion beam. Oftentimes one can boost the weak scattered signal far above the level of normal detection noise, thus offering exquisite detection sensitivities. Microscopies that harness this detection principle are described under the umbrella term "interferometric microscopies," and in particular iSCAT is a technique that has been developed to optimize the sensitivity for the detection of nanoparticulate matter.

We can express the interferometric detection of scattered light via the following equation:

$$
I_{\text {det }}=\left(\mathbf{E}_{\text {ref }}+\mathbf{E}_{\mathrm{sca}}\right)^{2}=\left|\mathbf{E}_{\text {ref }}\right|^{2}+\left|\mathbf{E}_{\mathrm{sca}}\right|^{2}+2\left|\mathbf{E}_{\mathrm{ref}}\right|\left|\mathbf{E}_{\mathrm{sca}}\right| \cos (\theta),
$$

where $I_{\text {det }}$, is the light incident upon our detector, $\mathbf{E}_{\text {sca }}$ the electric field of the scattered light of the sample and $\mathbf{E}_{\text {ref }}$ is the companion beam with which we interfere the scattered light. In practice this 

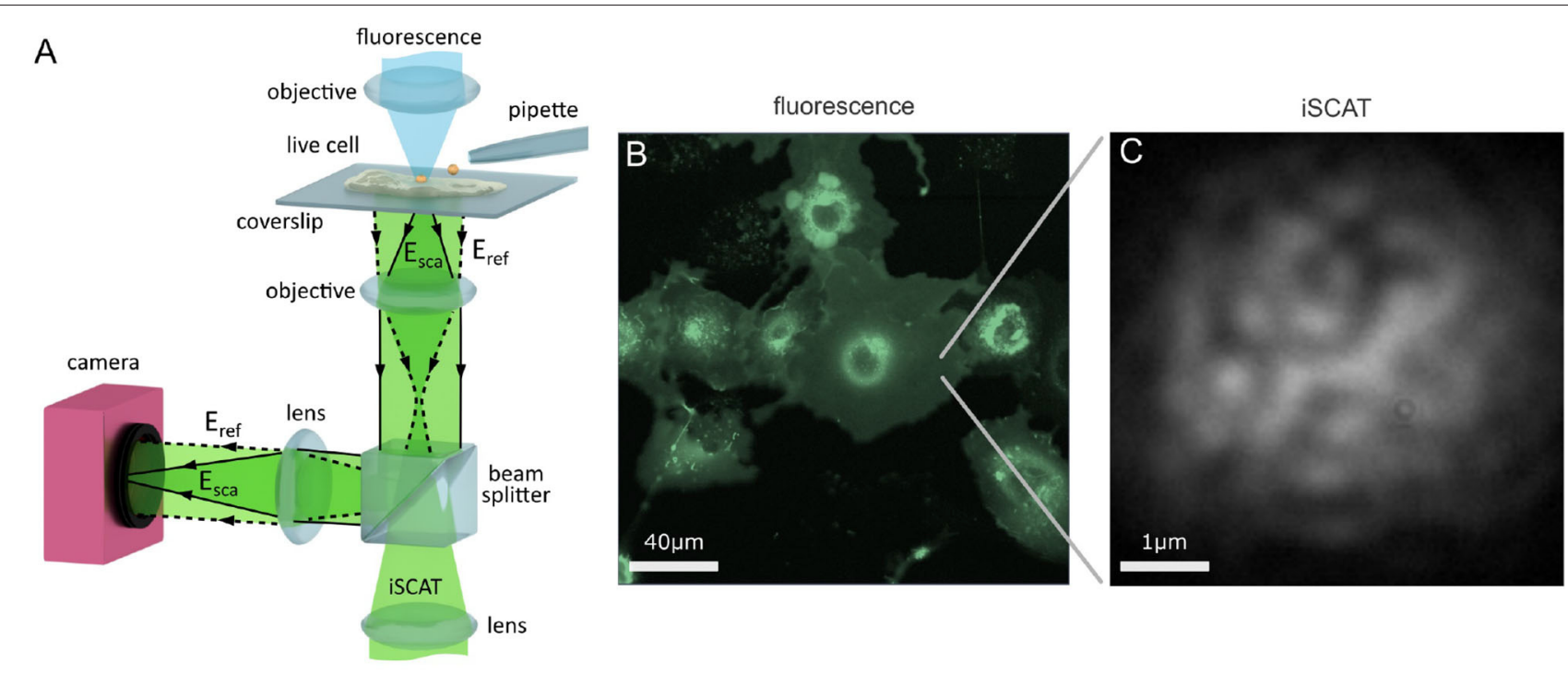

FIGURE 1 | Imaging the live cell with iSCAT microscopy. (A) Schematic of a wide-field reflection iSCAT microscope which incorporates a micropipette and a confocal fluorescence imaging channel. (B) A complementary macroscopic confocal fluorescence image of COS-7 cells transfected to over-express the EGFR-EGFP protein. (C) A raw widefield iSCAT image of the COS-7 membrane from the region highlighted in (B).

beam, referred to as the "reference" beam, can be obtained from a reflection of the illuminating beam in the optical path, which we discuss further in the following section. The key component of Equation (1) is the cross-term where one sees the multiplication of the scattered and reference fields to create a product with an overall larger intensity.

The phase term $\cos (\theta)$ determines the contrast of the interference term with respect to the bright signal of the reference intensity and the exact phase difference is determined by many contributions including the material of the scatter, the imaging arrangement and sample geometry (Mahmoodabadi et al., 2020).

\section{2. iSCAT Microscopy on the Live Cell}

A popular modality for iSCAT microscopy is the wide-field reflection arrangement shown in Figure 1A, where the sample is illuminated by a wide beam, and the reference beam (labeled $\mathbf{E}_{\text {ref }}$ ) with which the sample-scattered light $\left(\mathbf{E}_{\mathrm{sca}}\right)$ interferes originates from the portion of this incident illumination which back-reflects off the sample-bearing glass coverslip.

A key advantage of iSCAT microscopy is that the high sensitivity of detection permits imaging with excellent signal-tonoise levels even at extremely high speeds, with framerates in the range of 100-1,000,000 frames per second (fps) reported in the investigation of membrane diffusion (Lin et al., 2014; Spindler et al., 2016; Wu et al., 2016; Huang et al., 2017a; de Wit et al., 2018; Reina et al., 2018; Taylor et al., 2019). For imaging protein mobility on live cells, high framerates are particularly important in order to observe transient nanoscale molecular interactions which occur at swift milli- to microsecond time scales that may otherwise be not observable at lower framerates (Ritchie et al., 2005). Illumination intensities required for these high framerates are typically in the order of $\mathrm{I}=1-10 \mathrm{kWcm}^{-2}$, which are compatible with live cell imaging (Wäldchen et al., 2015).
In this work, we perform our investigations upon live HeLa and COS-7 cells. HeLa cells are an adherent, epithelial cell line derived from human cervix tissue and COS-7 cells are an adherent but fibroblast-like cell line that originate from the kidney tissue of the African Green Monkey. On HeLa, we track the endogenous EGFR whereas on COS-7 we track exogenous EGFR. In the latter case, this is achieved by transfecting the COS-7 cells with a plasmid encoding EGFR which increases the level of EGFR expressed within the cell. We link the EGFR plasmid with the enhanced green fluorescent protein (EGFP) to yield the fusion protein construct EGFR-EGFP which then allows us to visualize successful transfection through the fluorescence signal of EGFP. An asset of iSCAT microscopy is that it is fully compatible with fluorescence imaging (Kukura et al., 2009), and so one can incorporate conventional fluorescence microscopies and labeling strategies to assist with investigation on live cells.

We introduce a scanning confocal fluorescence channel from above the sample, shown in Figure 1A, to provide a macroscopic overview image of the live cell sample, as shown in Figure 1B. iSCAT microscopy may also be performed in parallel to confocal fluorescence imaging, and Figure 1C presents an image of a portion of the lower cell membrane viewed with iSCAT microscopy. The nature of iSCAT microscopy, particularly in this back-reflection scheme, produces interferometric images of the cell membrane that show a characteristic speckle pattern as shown in Figure 1C.

\subsection{On Labeling in iSPT}

The universality by which all materials scatter light results in their ability to be detected in iSCAT microscopy, provided appropriate care is taken to mitigate optical noise associated with the detection of light. In particular, it indeed has been shown to be possible with iSCAT microscopy to detect individual proteins 
A

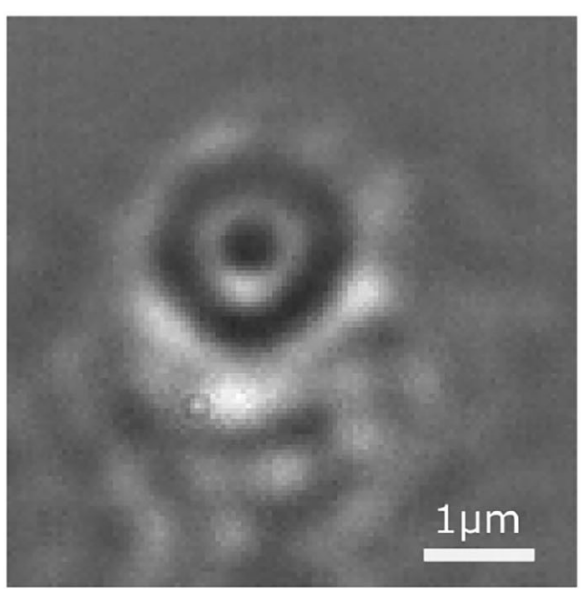

B

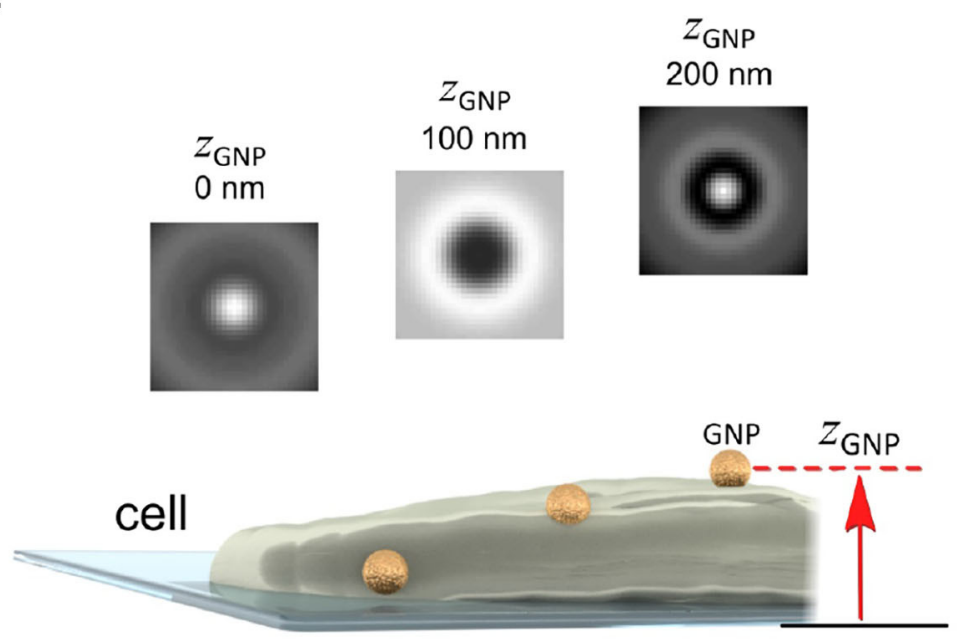

FIGURE 2 | Imaging and localizing a GNP probe in 3D with iSPT. (A) Background-corrected iSCAT image of the COS-7 cell membrane with a bound GNP probe (diameter $48 \mathrm{~nm}$ ). The GNP appears as a series of black and white rings from the iPSF. The distinctive ring pattern depends on the height of the GNP probe above the coverslip and also its position relative to the objective focus. (B) Schematic illustrating how the height of the GNP probe enables determination of its axial position: as the GNP diffuses on the membrane, a change in the height of the GNP above the coverslip produces a distinct iPSF which can be used to determine the height of the GNP $Z_{\text {GNP. }}$

directly without the need for any label when observed in isolation upon glass coverslips (Piliarik and Sandoghdar, 2014; McDonald et al., 2018; Young et al., 2018).

In tracking lipids and proteins when incorporated into a membrane, it is necessary to invoke use of a label to identify the molecule of interest and to render it distinct. Just as one can use a fluorescent label, one can equally use a strongly scattering nanoparticle as well as a label. Gold nanoparticles (GNPs) are widely used owing to their excellent scattering efficiency and bio-compatibility. As with fluorescence labeling, use of a colloidal label can potentially introduce artifacts that affect the diffusion under study, and these issues are addressed later in the discussion section.

\subsection{D Localization With iSPT Microscopy}

In iSPT, one images the light scattered by the GNP-labeled protein. However, since the remaining cell and its constituents also scatter light, they also produce a signal which we refer to as the imaging background. To localize the GNP-labeled protein in any given frame, it is first necessary to isolate this probe signal out of the imaging background.

Removal of the imaging background of the cell is, in general, challenging (Taylor and Sandoghdar, 2019). This is because firstly the scattered light has the same wavelength as the illumination and sample background and so simple wavelength-selective filtering is not possible. Secondly, the cell background is dynamic and fluctuating in nature, and consists of features with fine details of a similar size and shape to the probe signal. This dynamism stems from the fluctuating composition and morphology of the membrane and the cell interior, which is then observed as a random speckle-like pattern with temporal fluctuations in intensity, as illustrated in Figure 1C.
Several strategies have recently been reported which address this problem of removing the interferometric imaging background of the cell (Cheng and Hsieh, 2017; de Wit et al., 2018). One strategy exploits the difference between the highly symmetric circular pattern of the imaged gold probe and the "random" pattern of the cell background speckle (Taylor et al., 2019). Figure 2A presents an image of the plasma membrane of a COS-7 cell, where a single GNP has become bound to the membrane. As the nanoparticle is smaller than the optical diffraction limit, in imaging the GNP, especially interferometrically, one sees not an image of the particle but instead a series of rings of alternating contrast referred to as the interferometric point spread function (iPSF) (Mahmoodabadi et al., 2020). By using a circularlysymmetric feature extraction algorithm, the probe iPSF can be read from one frame of the video (Taylor et al., 2019; Mahmoodabadi et al., 2020).

The imaging background, however, should not necessarily be viewed as a nuisance, rather it can also provide useful information. For example, the interferometric image of the cell can indicate sample creep and drift which are important parameters to quantify and correct for in wanting to reliably interpret single particle trajectories (Taylor et al., 2019). Once the probe iPSF has been isolated from the background, one can then determine to nanometric precision the $3 \mathrm{D}$ position of the probe. The lateral $(x-y)$ position is found by determining the center of the symmetric iPSF, which can be accomplished through a variety of means such as by finding the center of symmetry (Parthasarathy, 2012), or through fitting of a twodimensional Gaussian intensity function or an interferometric diffraction-based model which describes the iPSF exactly (Mahmoodabadi et al., 2020). 


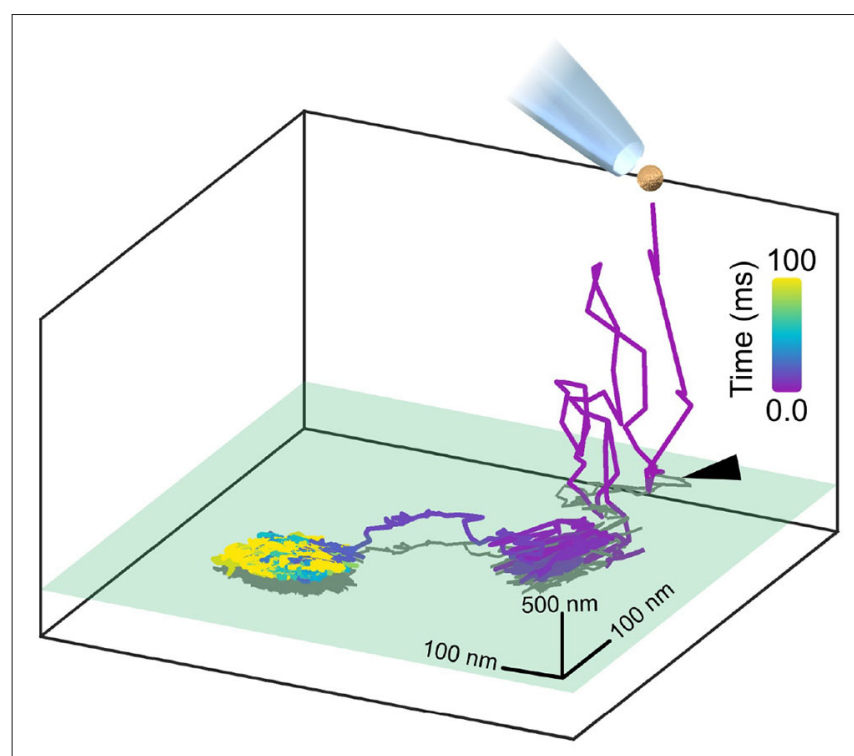

FIGURE 3 | Watching in situ the moment a GNP lands upon the plasma membrane. A GNP, once pipetted toward the COS-7 cell, is tracked axially over a range of $2 \mu \mathrm{m}$ whilst it diffuses in the culture medium before coming to land upon the cell membrane, with the initial point of contact with the membrane marked with a black arrow. The axial plane of the cell membrane is represented in green and a two-dimensional projection of the trajectory is plotted in gray. The whole landing event lasted approximately $100 \mathrm{~ms}$ and was recorded at 30,000 fps. The GNP used here was positively-charged so as to immediately and readily bind non-specifically to the negatively-charged cell membrane for the purpose of illustration.

To determine the height of the GNP above the coverslip $\left(z_{\mathrm{GNP}}\right)$, one exploits the information encoded within the alternating contrast of the iPSF ring pattern, which is a distinctive feature of iSPT (Mahmoodabadi et al., 2020). Figure 2B illustrates several iPSFs for a GNP that is diffusing over a region of the cell membrane which raises its height. The distinctiveness of the iPSF and the good sensitivity by which it can be measured enables nanometrically-precise lateral and axial localization. One can determine the relative change in height by direct calibration of the central contrast of the iPSF (Jacobsen et al., 2007), or by machine-learning assisted clustering of the iPSF (Taylor et al., 2019) or by direct fitting of the iPSFs to a model specifically describing iPSF formation (Mahmoodabadi et al., 2020).

\subsection{Watching in situ Landing of a GNP Probe on the Membrane With iSPT}

To provide an illustration of 3D nanometric iSPT of a GNP probe, we present Figure 3, wherein we track the landing of a GNP probe upon the cell membrane. To observe the labeling step is an important ability in interferometric particle tracking as one is able to ensure that indeed it is the GNP probe which is being observed but also one is able to know when the probe-protein interaction began which is especially important for investigating time-dependent processes.

Figure 3 demonstrates the in situ binding of a pipetted GNP probe to the plasma membrane, visualized through the
3D trajectory. Here in this example, for illustration we use a positively-charged GNP which readily binds to the negativelycharged plasma membrane. It should be noted that in this case, binding to the plasma membrane is purely electrostatic and not representing any particular lipid or protein motion in the membrane. Here, the GNP is tracked axially over a range of two microns where it is then seen to initially interact with the membrane, break loose before then ultimately becoming permanently attached.

\subsection{Describing the Erratic Mobility of Proteins}

If one watches the path taken by an individual protein as it navigates through the environment of the cell, one sees the protein takes steps that are seemingly random in length and direction. This behavior is driven by ever-present random fluctuations in the environment of the protein and is referred to as diffusion. When the mobility of a particle is driven by thermal fluctuations and otherwise unencumbered, we refer to the mobility of the particle as being Brownian or "normal."

When normal diffusion is enhanced or restricted by an external influence, for example, by a variety of geometric and steric interactions, then the diffusion becomes anomalous. Such anomalous diffusion encapsulates two regimes of mobility that are in many regards polar opposites. Anomalous diffusion, wherein the mobility is enhanced by the presence of phases of persistent motion, for example by the application of an external driving force, or complex velocity flow, is referred to as superdiffusion. Conversely, one can have sub-diffusion, where the dispersal process happens slower than in the case of normal diffusion, with a sub-linear scaling of the MSD (Montroll and Lebowitz, 1976; Bouchaud and Georges, 1990; Isichenko, 1992; Metzler and Klafter, 2000). In the plasma membrane, subdiffusion may arise from various biological mechanisms such as interactions with the organizational elements of the membrane, for example between cytoskeletal barriers, molecular crowding, or binding interactions between membrane molecules (Saxton, 1994, 1996; Jin and Verkman, 2007; Dix and Verkman, 2008; Jacobson et al., 2019). Determining the mechanistic origin of sub-diffusion in the cell is challenging, and remains an active area of research (Saxton and Jacobson, 1997; Saxton, 2012) with recent efforts presenting statistical frameworks to characterize and discriminate between different membrane models (Weber et al., 2012; Renner et al., 2017; Woringer et al., 2020).

\section{Mean Square Displacement Analysis of Membrane Mobility}

The general diffusive nature of membrane protein mobility implies that it is impossible to predict how the protein will proceed from one step to the next as the process is essentially random. Nonetheless, various statistical models have been proposed to interpret these random walks in order to describe any characteristic properties they may have (Metzler et al., 2014; Meroz and Sokolov, 2015). One immediate characterization is, for example, whether the walk exhibits normal or anomalous behavior, which may be an important indicator of function in the case of a transmembrane protein. 
One of the most widespread means of analysis is that of the Mean Square Displacement (MSD) where one examines the timedependent character of the diffusive walk to quantify whether the walk is normal or anomalous, and if it is anomalous, to which degree. We can express the MSD as:

$$
\operatorname{MSD}(\tau)=\Gamma_{\alpha} \tau^{\alpha}
$$

where $\tau$ represents an interval of time within the trajectory, $\Gamma_{\alpha}$ is a coefficient of proportionality and $\alpha$ is the temporal exponent (time-dependence) that we seek in order to quantify the nature of the diffusive walk. A protein undergoing unencumbered Brownian diffusion is characterized by a MSD which is linear in time, i.e., $\alpha=1$, which is so-called "normal" diffusion and $\Gamma_{\alpha=1}=D$ is just the diffusion constant. For a diffusing protein that is not truly free, i.e., being subject to additional influences beyond thermal fluctuations, then the diffusion can be anomalous and $\alpha \neq 1$. An $\alpha>1$ describes super-diffusion, and occurs, for example, in instances where the protein is being specifically guided along a certain path such as which occurs in intracellular transport. An $\alpha<1$ denotes the hindered mobility of subdiffusion, and for diffusion measured in the plasma membrane a typical value of $\alpha=0.7$ is characteristic by conventional ensemble MSD analysis (Saxton and Jacobson, 1997). With careful analysis of the MSD and temporal exponent, one can hence characterize the diffusive character of the membrane protein within the local membrane environment.

To compute the MSD, one calculates how the average distance walked squared for a given interval of time depends on how long the time interval is. To obtain a reliable measure of the time-dependence of the walk one requires a sufficient number of recordings to give an average that is statistically robust (Kepten et al., 2013, 2015; Vestergaard, 2016). One method is to build an average out of many similar trajectories from separate measurements. Alternatively one may take a single sufficiently long trajectory and subdivide it into shorter pieces which are then averaged together. The former approach is known as a ensemble average and the latter is referred to as a time-average. A diffusive system in which these two approaches are equivalent is said to be ergodic. The plasma membrane of the cell is an example of a system that can exhibit weak ergodicity breaking which is a behavior chiefly originating out of inhomogenous confinement and stalling of the diffusing protein in the membrane (Metzler et al., 2014). As a consequence, these two approaches can not generally be assumed to be equivalent.

In our work presented here, we accumulate a sufficient number of frames from a very short window in time, typically representing just a small fragment of the complete trajectory. We then roll the MSD analysis incrementally across the whole trajectory and thus build up a measure of how the temporal exponent is changing throughout the trajectory. Doing so allows one to visualize how the mobility is evolving in time as the diffusing protein encounters new and differing obstacles and environments (Taylor et al., 2019). This contrasts with conventional MSD analysis where one considers all points in a trajectory at once (see Supplementary Material 1).
The rolling window time-averaged MSD is given by:

$$
\operatorname{MSD}_{i}(\tau)=\left\langle\left(r\left(t_{j}+\tau\right)-r\left(t_{j}\right)\right)^{2}\right\rangle_{i}=\Gamma_{\alpha_{i}} \tau^{\alpha_{i}}+\sigma_{x y}
$$

where $i$ denotes the index of the temporal window wherein the MSD is to be computed, $r\left(t_{j}\right)$ is the $x-y$ position of the protein at a time $t_{j}$, where $t_{j}$ represents points in time belonging within the window and $\langle\ldots\rangle$ denotes the calculation of an average. The temporal exponent which characterizes the time-dependence of the MSD within the given window $i$ is denoted by $\alpha_{i}$.

The microsecond imaging resolution of iSPT allows one to quantify the time-dependence of the protein mobility to millisecond resolution when employing the rolling time-average. One, however, must also be careful when analysing trajectories recorded at high-frame rates. Firstly, in general, one must always account for the error introduced by the localization precision for each specific trajectory point $\left(\sigma_{x y}\right)$ in the computation of the MSD as an omission of this uncertainty would lead to underestimation of the respective temporal exponent (Martin et al., 2002). When imaging at high framerates in particular, it is possible that the step taken by the protein between two pairs of frames is comparable to, if not smaller than, the certainty with which the start and end positions are able to be known. In such cases, when performing the analysis one must be careful to avoid use of these frame pairs to avoid erroneous calculation of $\alpha_{i}$.

\section{Directional Correlation Analysis}

A complementary means to quantitatively identify the presence of obstacles and barriers to diffusion or directed transport is to look for correlation in the direction in which any two steps are taken. In the case of free diffusion, one would expect no correlation in the walked trajectory as the process is essentially random with no memory. In the presence of obstacles, however, one would expect the occurrence of recoil or "knock-back" upon collision with said obstacles. In the case of directional transport, one would expect a prevalence of a certain direction, i.e., a positive correlation. Directional correlation analysis seeks to quantify the occurrence of such collisions by considering the angular correlation between two steps, and seeing if this is a meaningfully repeated occurrence.

The directional correlation is calculated by first considering two steps taken by the diffusing probe. The first step, $\Delta \vec{r}_{1}$, is the vectorial displacement between two frames separated in time by $\tau: \Delta \vec{r}_{1}=r(t+\tau)-r(t)$, where here we take $\tau=5$ frames. The second step begins where step $\Delta \vec{r}_{1}$ ended, and also occurs over an equal time interval, i.e., $\Delta \vec{r}_{2}=r(t+2 \tau)-r(t+\tau)$. The cosine of the angle between these two steps is then computed, thus giving an expression for their angular correlation. As with the MSD, this calculation is repeated for all pairs of steps that occur within a smaller window $(i)$ of the whole trajectory so that changes in the directional correlation can be identified over the course of the trajectory. Formally, we calculate the directional correlation $C_{i}$ for the vectorial steps $\Delta \vec{r}_{1}$ and $\Delta \vec{r}_{2}$ via the following (Weeks and Weitz, 2002; Munder et al., 2016):

$$
C_{i}=\left\langle\frac{\Delta \overrightarrow{r_{1}}}{\left|\Delta \overrightarrow{r_{1}}\right|} \cdot \frac{\Delta \overrightarrow{r_{2}}}{\left|\Delta \overrightarrow{r_{2}}\right|}\right\rangle_{i}
$$


where $|\ldots|$ denotes the magnitude of the vectorial step and as before, the average is computed for all pairs in the $i^{\text {th }}$ window. A truly random process possesses no overall angular correlation between any pairs of steps, and so $C=0$. A positive directional correlation would imply the diffusing probe exhibits some persistent motion and a negative correlation suggests persistent knock-back or deflection.

\section{TRACKING EGFR ON THE LIVE CELL WITH iSPT}

\subsection{Labeling the Receptor}

In our investigation to label the EGFR, we functionalize GNPs of $48 \mathrm{~nm}$ diameter with EGF to produce an EGF-GNP probe that specifically binds to the EGFR. To do so, we use streptavidincoated GNPs and EGF probes with attached biotin molecules. The strong chemical bond between streptavidin and biotin serves as the linker between the GNP and the EGF ligand. A micropipette is then used to deliver small quantities of the EGFGNPs to the immediate vicinity of the cell under investigation. This introduces the distinct advantage of setting a clear start point in which the probe is known to begin to interact with the membrane, which is important for biological processes stimulated by probe binding. The permanence of the scattering signal from the GNP probe along with the ability of iSPT to track nanoparticles in $3 \mathrm{D}$ over an extended axial range permits observation of the labeling process prior to immediate tracking of the mobility of the labeled protein. Once the functionalized GNP has bound the receptor we refer to this complex as "EGFR-GNP."

\subsection{Anomalous Mobility on the Plasma Membrane}

In Figure 4, we present an example of a typical trajectory following the labeling of the EGFR with an EGF-GNP probe. This trajectory was recorded on the lamellapodium of a COS7 cell at a framerate of 30,000 frames per second for a duration of $8.3 \mathrm{~s}$ (250,000 trajectory points). The $x-y$ projection of the trajectory is plotted in Figure 4A. The axial information of the trajectory reveals diffusion on a locally-flat but slowly curving surface expected from the lamellapodium, depicted in Figure 4B.

The extended period of observation shows the frequent occurrence of the probe revisiting the same regions of the membrane as well as navigating through distinct patches with differing sparsity of trajectory points. One must express care in interpreting the trajectories by visual inspection, and thus we defer to quantification by MSD and directional correlation analysis.

We first begin by calculating the whole-trajectory MSD, shown in Figure 4C. When presented on a log-log scale, shown in the inset of Figure 4C, the plot becomes linear and the gradient gives the temporal exponent which typifies the whole trajectory. By using the rolling-window MSD we are able to explore the "microscopic" changes in the diffusional character of the trajectory in time. The temporal exponent of diffusion is plotted in Figures 4D,E. Additional information on the determination of $\alpha_{i}$ is presented in Supplementary Material 2. Here we use a rolling window of length of 1,000 frames, corresponding to a window with temporal duration $T_{\mathrm{w}}=33 \mathrm{~ms}$, and find the temporal exponent to be fairly robust against the size of the window length, shown in Supplementary Material 3. Generally, the trajectory has a global mean value of $\alpha_{i}=0.7 \pm 0.1$ confirming indeed the diffusion is anomalous. Inspection of the rolling value reveals a distribution in the value of $\alpha_{i}$, which appears to be region-specific, with some portions of the walk encountering strong confinement with $\alpha_{i} \approx 0.5$. In comparing the directional correlation, Figures $4 F, G$, to their respective temporal correlation partner, we find strong agreement between both methods of analysis with the same temporal fluctuations and spatial distribution evident in both. The mean directional correlation value for the trajectory, $C=-0.2 \pm 0.1$ implies deflection and knock-back of varying strength. In tandem, both $\alpha$ and $C$ suggest that the sub-diffusion occurring in the trajectory results from the obstructed random walk of the EGFR-GNP, and that this interaction is locally heterogeneous.

The origin for the heterogeneous environment encountered by the EGFR protein-which manifests in the anomalous trajectory of the protein-is multifold. Whilst it is beyond the scope of this work to give a comprehensive account of all the known means of interaction, it is nonetheless interesting to consider some of the core principles of membrane organization that readily affect EGFR. To that end we might begin with the role played by the substructure and compartmentalization of the plasma membrane itself. Kusumi and co-workers first proposed that the membrane is partitioned into compartments, sometimes doubly so, by the underlying actin cytoskeleton which thus acts as a diffusive barrier to membrane proteins, with compartment side lengths in the range $L=40-800 \mathrm{~nm}$ depending on the cell type (Kusumi et al., 1993, 2005, 2012; Fujiwara et al., 2002, 2016). These compartments, which frustrate free diffusion, appear to render useful biological function. For example, monomeric proteins tend to be able to pass the compartment boundaries more easily via a process known as "hopping" or "hop-diffusion," whereas dimeric or oligomeric forms of the protein tend to remain confined (Kusumi et al., 2012). As a result, such compartments may enrich or exclude multimeric proteins and thus constitute functional platforms in e.g., signaling or adhesion.

It is interesting to reflect upon the fact that our understanding of the important role membrane compartmentalization plays upon diffusion was borne out of the early pioneering work of Kusumi and co-workers more than two decades ago. By using one of the earliest realizations of iSPT microscopy which granted them sufficient temporal resolution, membrane compartmentalization, and "hop-diffusion" could be inferred through careful analysis of the trajectories of membrane proteins. With the recent progress in improved detection sensitivity, spatio-temporal resolution, and precision from the everexpanding iSCAT and iSPT microscopy community (Piliarik and Sandoghdar, 2014; Huang et al., 2017a; Young et al., 2018; Taylor et al., 2019), it is an exciting prospect to revisit and explore the role membrane compartmentalization plays on protein mobility with renewed experimental and theoretical vigor (Lyman et al., 2018). 


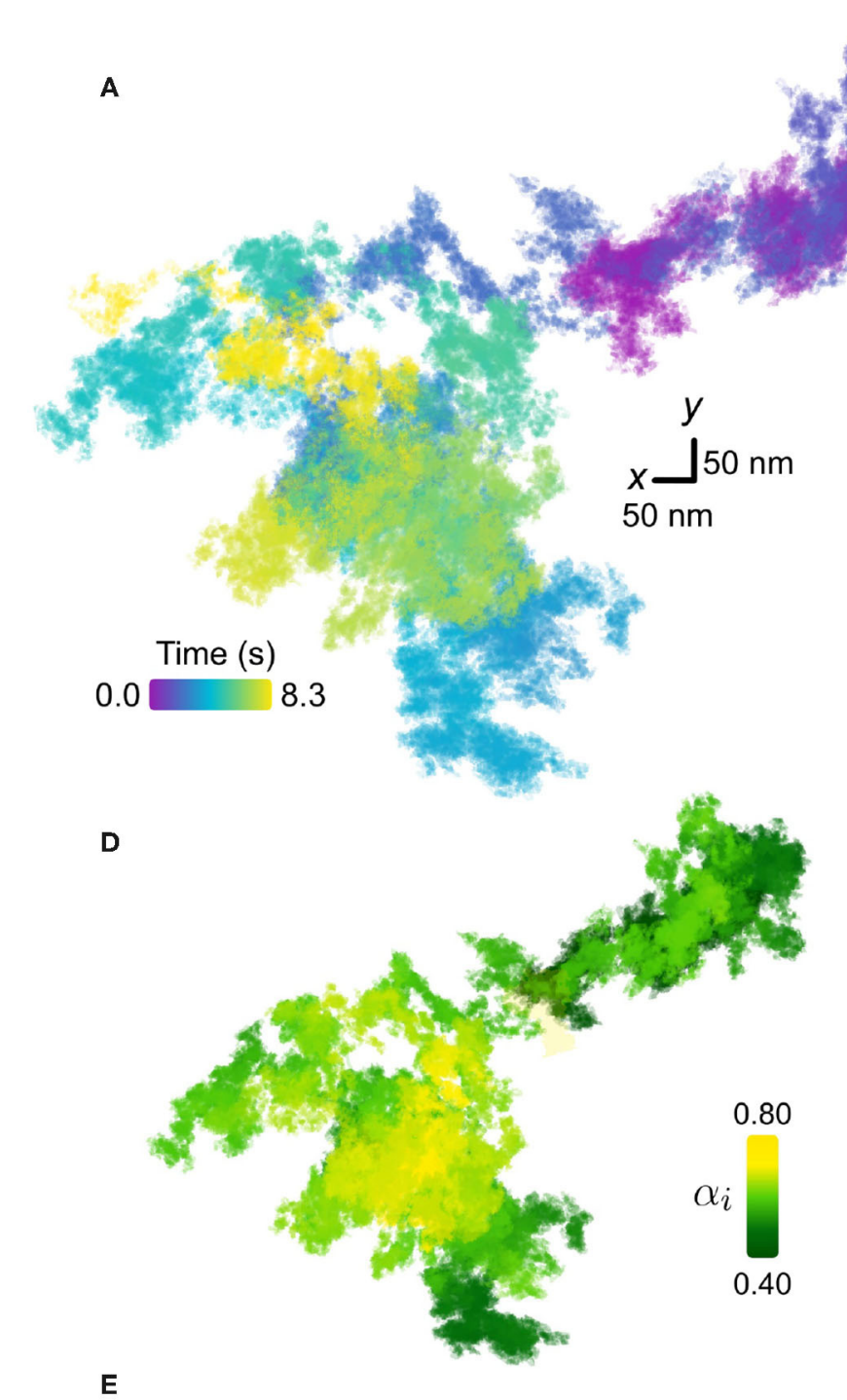

Temporal exponent

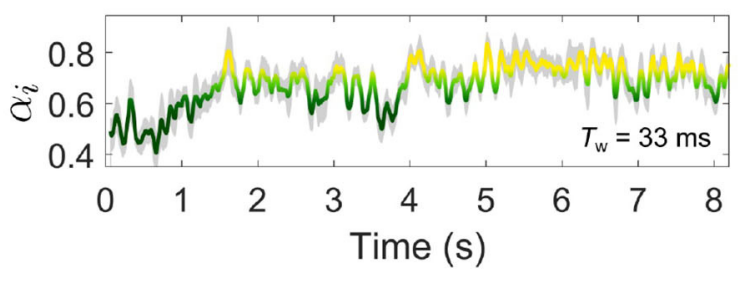

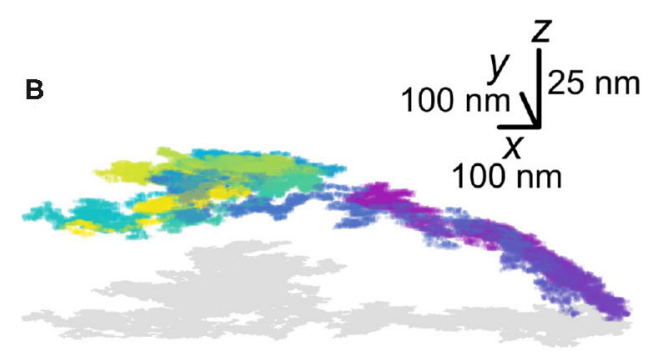

C
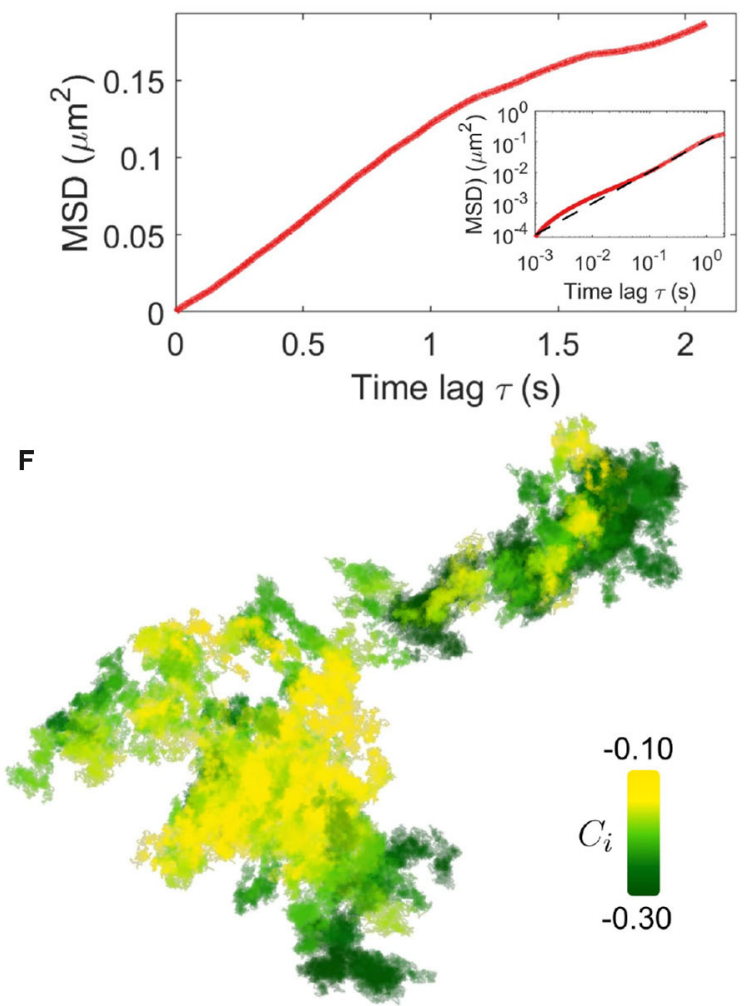

G

\section{Directional correlation}

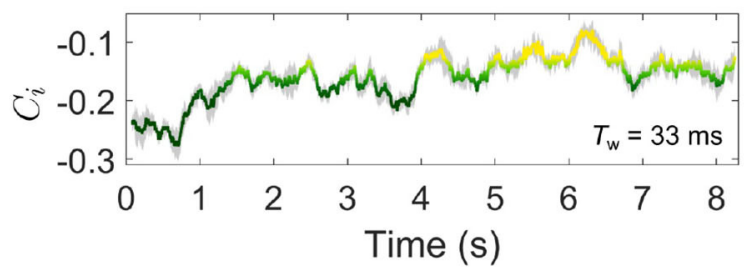

FIGURE 4 | Anomalous EGFR diffusion in the plasma membrane revealed by high-speed iSPT. (A) A typical EGFR-GNP trajectory recorded with 30,000 frames per second for a duration of $8.3 \mathrm{~s}$ on a COS-7, here shown as $x-y$ plane. (B) The trajectory from (A) viewed in 3D, revealing global curvature. The 2D projection is depicted in gray. (C) The whole-trajectory MSD. Inset shows the same plot but on a log-log scale with a linear slope as a guide to the eye. (D) The rolling temporal exponent of diffusion $\alpha_{i}$ for the trajectory for an incrementally sliding window of 1,000 frames ( $\left.T_{\mathrm{w}}=33 \mathrm{~ms}\right)$, represented by the color scale, mapped onto the $x-y$ trajectory. (E) The temporal exponent plotted as a function of time. (F) Similar to (D), the rolling directional correlation $C_{i}$ is mapped onto the $x-y$ trajectory and also expressed as a function of time (G). The gray shadow in $\mathbf{( E , G ) ~ s h o w ~ t h e ~ b o u n d s ~ o f ~ u n c e r t a i n t y ~ i n ~ c a l c u l a t i o n ~ o f ~ e a c h ~ r e s p e c t i v e ~ q u a n t i t y . ~}$

We next consider an additional example of membrane diffusion similar to Figure 4, shown in Figure 5, where the lateral trajectory (Figure 5A) shows again a heterogeneity and sparsity in the walked path as well as clear re-visiting of domains as opposed to the continued visiting of new membrane areas. Performing the MSD (Figures 5B,C) and directional correlation 


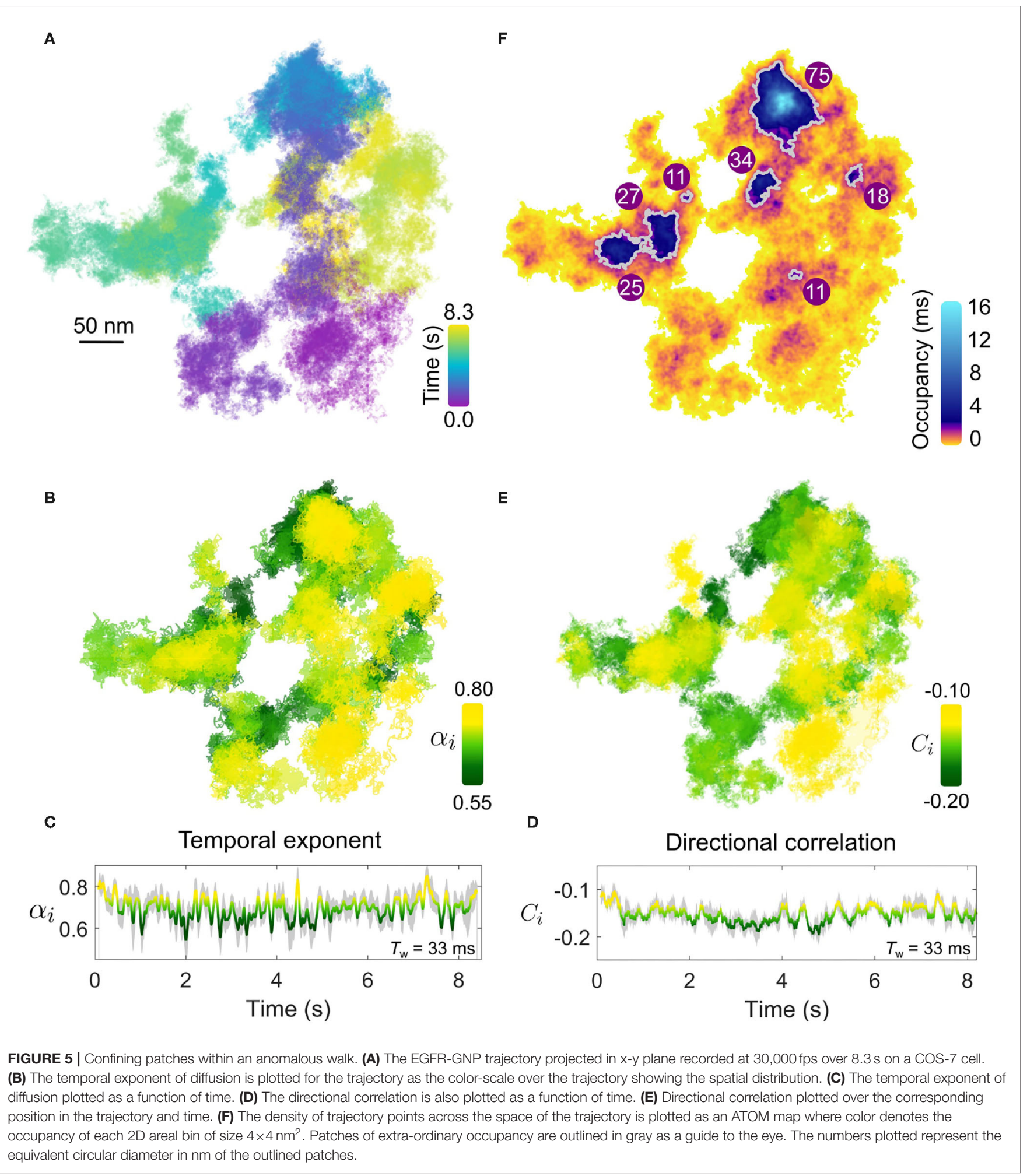

analysis (Figures 5D,E) upon this trajectory for again a window of 1,000 frames ( $T_{\mathrm{w}}=33 \mathrm{~ms}$ ) reveals interesting features. Firstly, we observe again strong heterogeneity in $\alpha$ and $C$ throughout the trajectory that is region-specific but not necessarily always the same through the course of time of the measurement. In particular, the temporal exponent shows a strong variance in value but generally is centered around $\alpha=0.7$. Interestingly, the directional correlation shows general agreement in trend with 


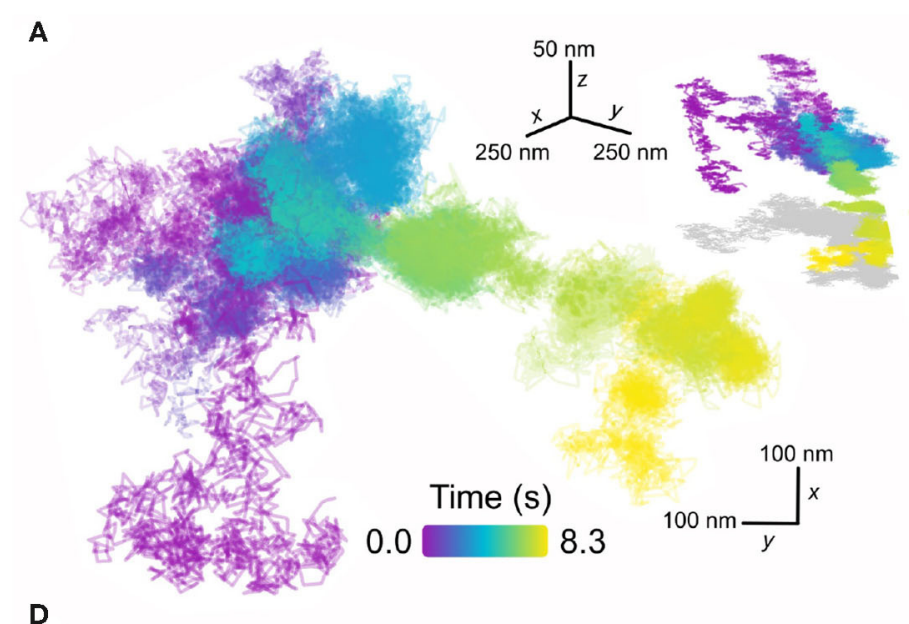

D

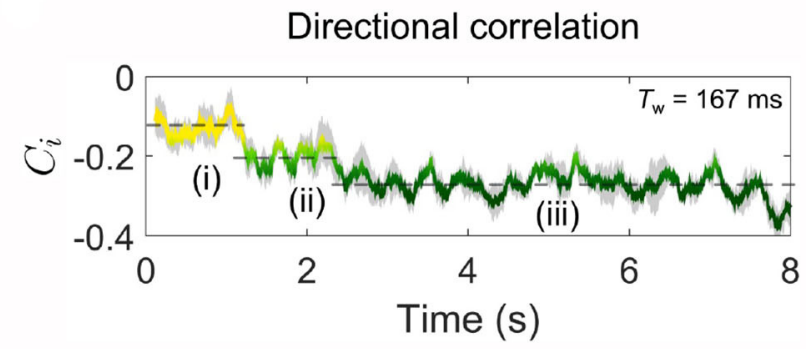

B

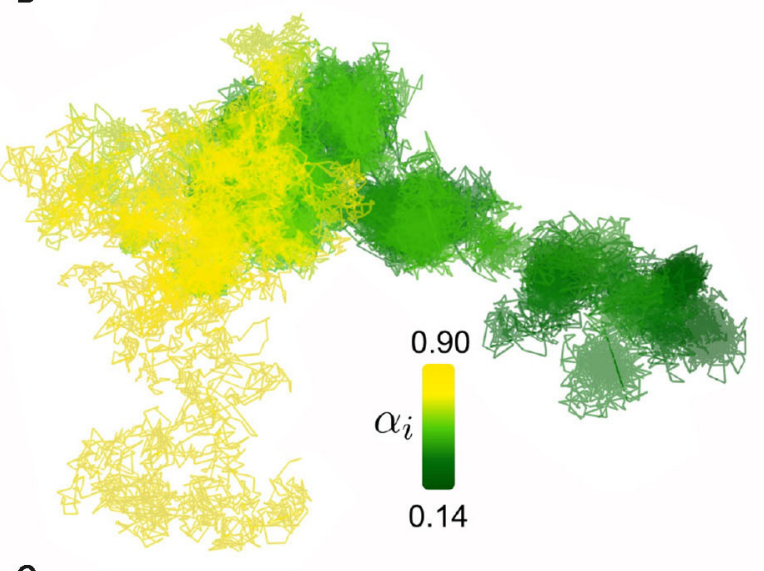

Temporal exponent

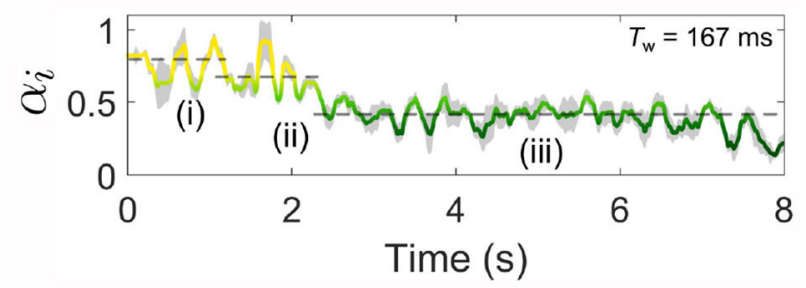

FIGURE 6 | The endurance of iSPT imaging enables visualization of dynamic, time-evolving processes. (A) An exemplar trajectory of a EGFR-GNP on a HeLa cell is plotted for a measurement over $8.3 \mathrm{~s}$ recorded at $6,000 \mathrm{fps}$. Inset: the 3D trajectory of the $x$-y projection shown in (A), with a 2D "shadow" projection plotted in gray. (B) The temporal exponent of the trajectory presented in (A) plotted spatially over the trajectory positions. (C) Temporal exponent plotted as an explicit function of time. (D) The corresponding directional correlation of the trajectory is also plotted as a function of time. Both temporal exponent and directional correlation use a sliding window of 1,000 frames $\left(T_{w}=167 \mathrm{~ms}\right.$ ) and the dashed lines guide the eye to the three distinct phases of diffusive behavior, labeled (i)-(iii).

the temporal exponent, and is also suggesting "knock-back." But unlike the example of Figure 4, there is weaker correspondence between the sharp changes in the degree of sub-diffusion and the step correlations. Since these two forms of analysis probe different aspects of the EGFR-GNP trajectory this is entirely plausible. One possible interpretation is that the labeled EGFRprotein is slowed down within a "sticky" sub-domain because then the distance traveled in time, quantified by $\alpha_{i}$, would show sub-diffusion, whilst if no immediate barrier or boundary is encountered no correlation in steps would be evident. Here we define sticky to mean orders of magnitude longer residency than typically observed from the surrounding membrane, here longer than $1 \mathrm{~ms}$.

Furthermore, visual inspection also reveals the presence of patch domains consisting of a higher density of trajectory points. The presence of nanodomains on the membrane, where one believes that diffusion of a transmembrane protein becomes transiently arrested, is of great interest. The high temporal resolution of iSPT permits us to visualize the presence of domains where the EGFR-GNP becomes confined by simply dividing the $x-y$ trajectory into $2 \mathrm{D}$ bins and counting the occupancy of each bin. Such a plot we refer to as the accumulated total occupancy map (ATOM) (Taylor et al., 2019). Confining domains would consist of a higher population of trajectory points than non-confining, sparse regions. Figure $5 \mathrm{~F}$ presents the ATOM plot for the trajectory for an areal bin size of $4 \times 4 \mathrm{~nm}^{2}$. Here we use a highly non-linear color scheme to denote the occupancy to elucidate the wide range in residence times. We calculate the residence time by multiplying the total number of trajectory points per bin by the exposure time of one frame. The ATOM plot of Figure 5F displays several clear patches of extended residency with a mean equivalent circular diameter in nanometers illustrated on each prominent patch outlined in gray. Such regions of extended residency might represent favorable biochemical, structural, or morphological features of the cell membrane, although lacking further information one can only speculate on the nature of such features.

One advantage of iSPT for investigating membrane processes is the ability to observe the EGFR-GNP for an extended, in principle indefinite, period owing to the absence of photodegradation of the GNP probe. Coupled with the high temporal resolution and the sliding window analysis one can therefore monitor cellular processes in which the EGFR-GNP is involved, as reflected through marked changes in its diffusional behavior.

Figure 6 presents such an example for diffusion occurring on the plasma membrane. Inspecting the lateral trajectory Figure 6A reveals an apparent change in the diffusion which in time becomes progressively more spatially confined, with 
eventually a concerted directional motion replacing the randomlike exploration of the membrane. Examining the $3 \mathrm{D}$ projection of the trajectory (shown in the inset of Figure 6A), one sees the latter directed phase of the diffusion carries the EGFR-GNP down and away from the region of initial confinement. These distinct changes also manifest clearly in the kinetic parameters of the trajectory. For example, the temporal exponent (Figures 6B,C) confirms a stark transition from a nearly-free like diffusion $\left(\alpha_{i}=0.9\right)$ in the first several seconds of observation that quickly transitions into a strongly confined mode $\left(\alpha_{i}=0.4\right)$ that is maintained until the end of observation. Our sliding window analysis explores the diffusive nature to time lags bounded within the window length, here $T_{\mathrm{w}}=167 \mathrm{~ms}$, and thus we discover that within this phase the EGFR-GNP is strongly confined. These observations are similarly reflected in the directional correlation which displays persistent recoil from a confining obstacle and identifies three stages of the trajectories evolution, marked (i)-(iii).

Such a change in the diffusional behavior of EGFR shown here is evocative of processes such as the binding of the EGFR-EGF complex to the actin skeleton beneath the membrane. The actinbinding domain located on the EGFR (Den Hartigh et al., 1992) can guide association with the cytoskeleton. This interaction has been shown to lead to an oriented transport by the flow of the skeleton accompanied by a decrease in diffusion (de Bruin et al., 2007), since remodeling of cortical actin is an active and important organizational motif for membrane molecules (Gowrishankar et al., 2012).

\subsection{Confined Diffusion on the Plasma Membrane}

Aside from sub-diffusion which still explores sufficiently large regions of the membrane, another mode observed is a diffusion where the probe moves very little from its original position and thus is often classified as being confined. These modes of diffusion are also interesting to investigate since restriction in the mobility of a membrane protein is an especially important aspect of membrane organization and a means to regulate function of signaling proteins such as EGFR.

In Figure 7, we present two examples of diffusion which is markedly confined within a spatial domain of approximately $100 \times 100 \mathrm{~nm}^{2}$. The confined nature of the diffusion is most visually apparent when one compares the lateral trajectories of Figures 7A,D with that of Figure 4A which was recorded for the same duration and framerate. The temporal exponent of the diffusion, shown in Figures 7B,E for a sliding window length of 1,000 frames $\left(T_{w}=33 \mathrm{~ms}\right)$ similarly confirms the strong confinement of the EGFR with a consistent average value of $\alpha=$ 0.6 observed for both confined examples, although the trajectory of Figure 7A shows slightly larger variance.

Here, in this example the diffusion occurs within a limited spatial region and within the apparent boundary of this region the entire space is repeatedly explored by the EGFR-GNP. This then opens the interesting possibility to examine the spatial occupancy of the trajectory through the ATOM plot wherein the frequent sampling of the membrane environment through our high-resolution tracking might reveal information about any structures influencing the EGFR-GNP diffusion. The ATOM plots for trajectories shown in Figures 7A,D are plotted in Figures 7C,F, respectively for an areal $2 \mathrm{D}$ bin of $4 \times 4 \mathrm{~nm}^{2}$. Inspection of the occupancy elucidated in the ATOM maps reveals distinct patches and heterogeneous patches of particularly long confinement. In Figure $7 \mathbf{B}$, we identify a patch of $\approx 25 \mathrm{~nm}$ diameter as well as intermediate structures, typically located centrally, with also circular-like structures. In Figure 7F, we find numerous patches of extended occupation which are not homogeneous, but appear perforated with holes, suggesting regions of partial exclusion. Inspection also suggests the presence of circular-structures of a similar mean size of $25 \mathrm{~nm}$, possibly pointing toward structures in the membrane the EGFR-GNP is interacting with whilst confined within the corral.

Another important example of confinement of a diffusing protein which occurs on the plasma membrane is that of confinement into "pits" or "bowls" that are often associated with endocytosis of membrane proteins. For example, it is known that one route for EGFR internalization is through clathrin-mediated endocytosis, where clathrin assembles a bowl-like structure to envelope the portion of the membrane to be internalized.

In Figures 8A,B, we present an example of an EGFR-GNP trajectory, seen from two perspectives, recorded at 20,000 fps over $1.0 \mathrm{~s}$, and a second example in Figure 8C recorded at 30,000 fps over $3.2 \mathrm{~s}$. Both examples show a 3D trajectory which reveals the EGFR-GNP is confined to the surface of a $3 \mathrm{D}$ bowl, with both examples presenting a bowl with an approximate diameter of $350 \mathrm{~nm}$. The bowl morphology is most apparent when one interpolates through all the trajectory points to render an effective smooth surface, shown in Figure 8D.

In previous work, we observed the trajectory of the EGFRGNP within such a bowl that suggested a persistent rotation of the probe around the center of mass of the bowl. We denote the angular position of the probe with $\phi$, shown schematically in Figure $8 \mathrm{D}$, and plot the angular position as a function of time for the trajectories of Figures 8A,C in Figures 8E,F, respectively. In these angular plots, the radial extent denotes time, and in doing so one sees a back and forth rotation in Figure 8E, as reported previously (Taylor et al., 2019). It should be noted however, that not all bowl-like confinements necessarily exhibit this clear and persistent rotational motion. Figure $\mathbf{8 F}$ presents an example where no coherent rotational motion is seen, instead the probe was able to diffuse more erratically about the entire surface, but with occupancy favoring the lower regions of the bowl.

Given that EGFR is known to be internalized via clathrin mediated endocytosis and the similarity these EGFR-GNP bowltrajectories bear with such pits, a potential biological origin is identified. It stands as an exciting line of future inquiry, however, to affirm the role these pits play in the EGFR membrane biology. Furthermore, one can identify whether the EGFR-GNP probe is mobile within a static membrane bowl, or whether the probe is bound to the membrane and the whole bowl itself undergoes rotation or alternatively whether a combination of the two is at play. Nonetheless, these measurements demonstrate the potential for iSPT to provide new avenues for deeper nanoscopic insight into established membrane biology. 
A

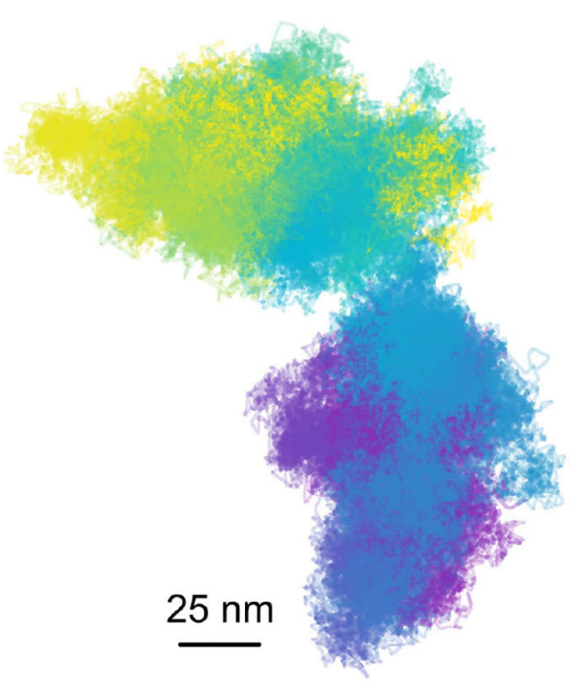

B

Temporal exponent

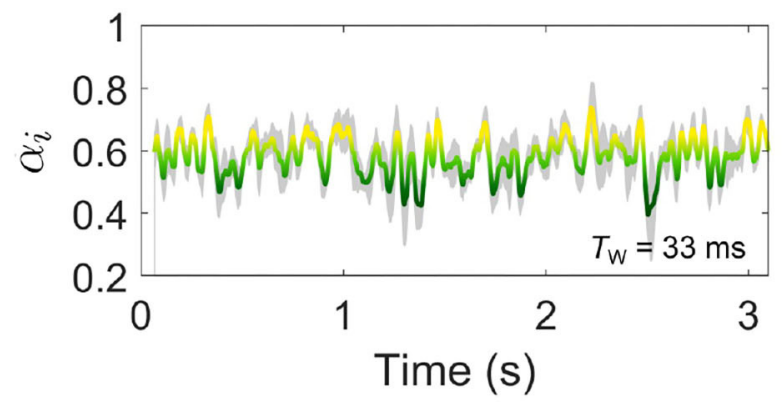

C

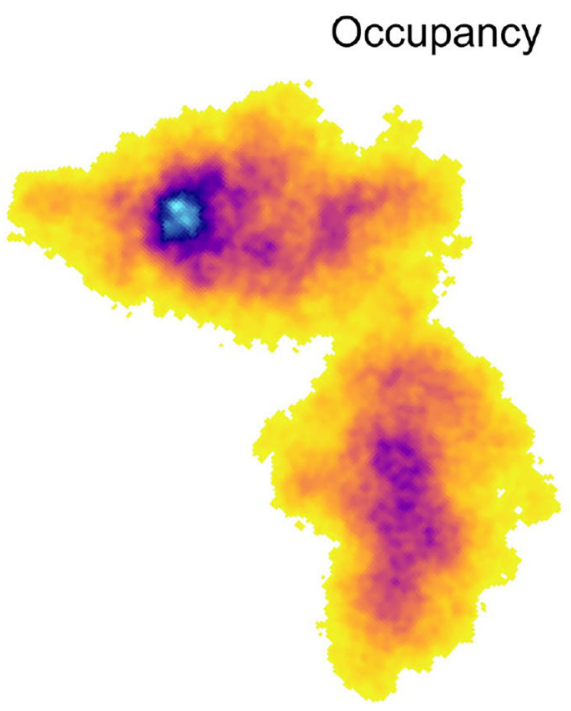

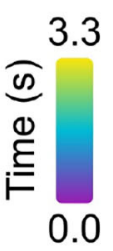

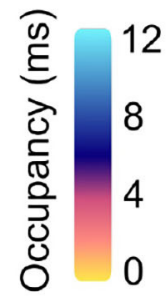

D

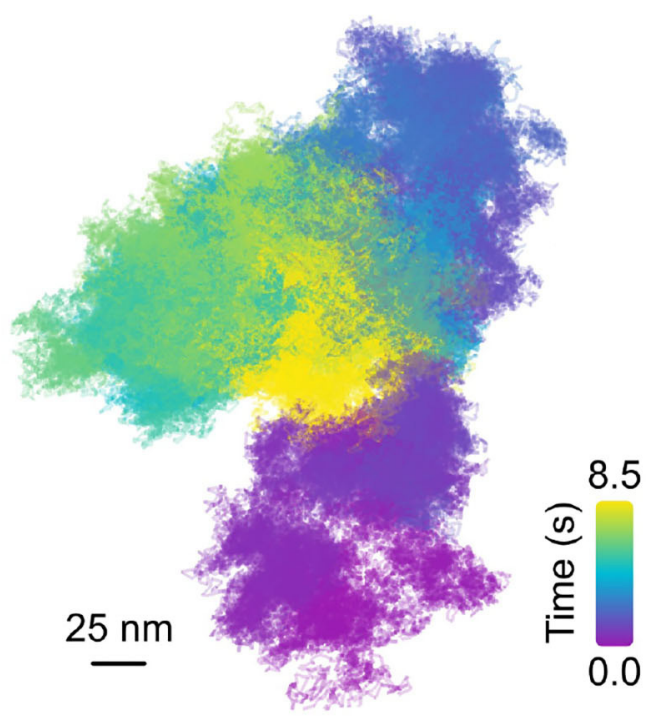

E

\section{Temporal exponent}

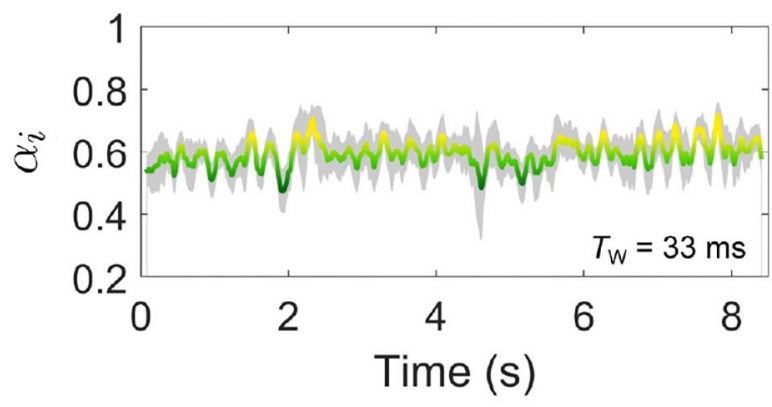

$\mathbf{F}$

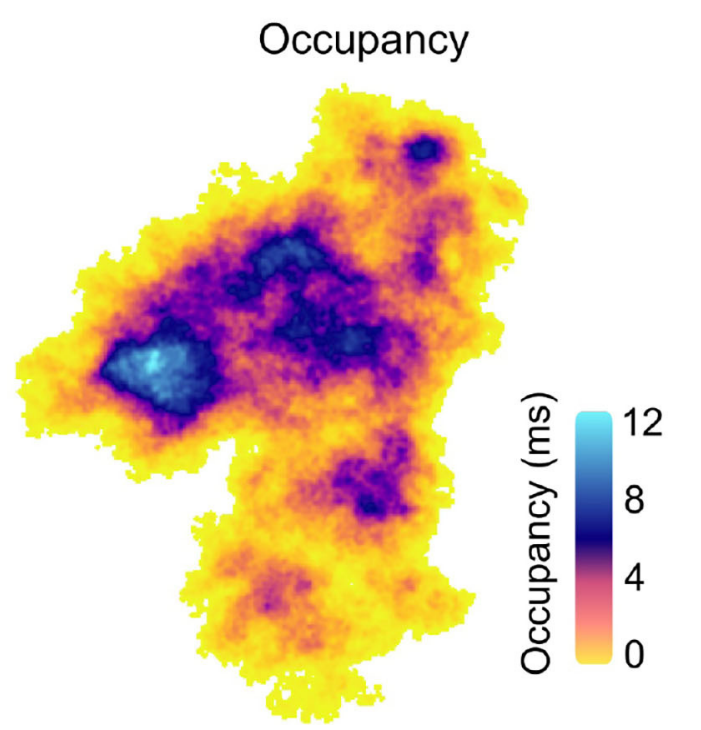

FIGURE 7 | Confinement on the plasma membrane of a COS-7 cell. (A) The trajectory for an example of confined EGFR-GNP diffusion on a COS-7 cell is presented. The imaging framerate of $30,000 \mathrm{fps}$ for a total of $3.3 \mathrm{~s}$ gives a total of 100,000 trajectory points. (B) The temporal exponent for the trajectory in (A) with an averaging window of $T_{\mathrm{w}}=33 \mathrm{~ms}$. (C) The respective ATOM plot for the trajectory of (A) is presented in (C). The areal bin size is $4 \times 4 \mathrm{~nm}^{2}$ and patches of extra-ordinary residence are reflected in the non-uniform color scale encoding total occupation time. (D) The trajectory for a second example of confined membrane diffusion, recorded at the same framerate as (A), but for $8.5 \mathrm{~s}$, giving a total of 250,000 recordings. (E) The corresponding temporal exponent for the trajectory in (D) with an averaging window of $T_{w}=33 \mathrm{~ms}$. (F) The ATOM plot for the trajectory of (D), with an areal bin size is $4 \times 4 \mathrm{~nm}^{2}$. 


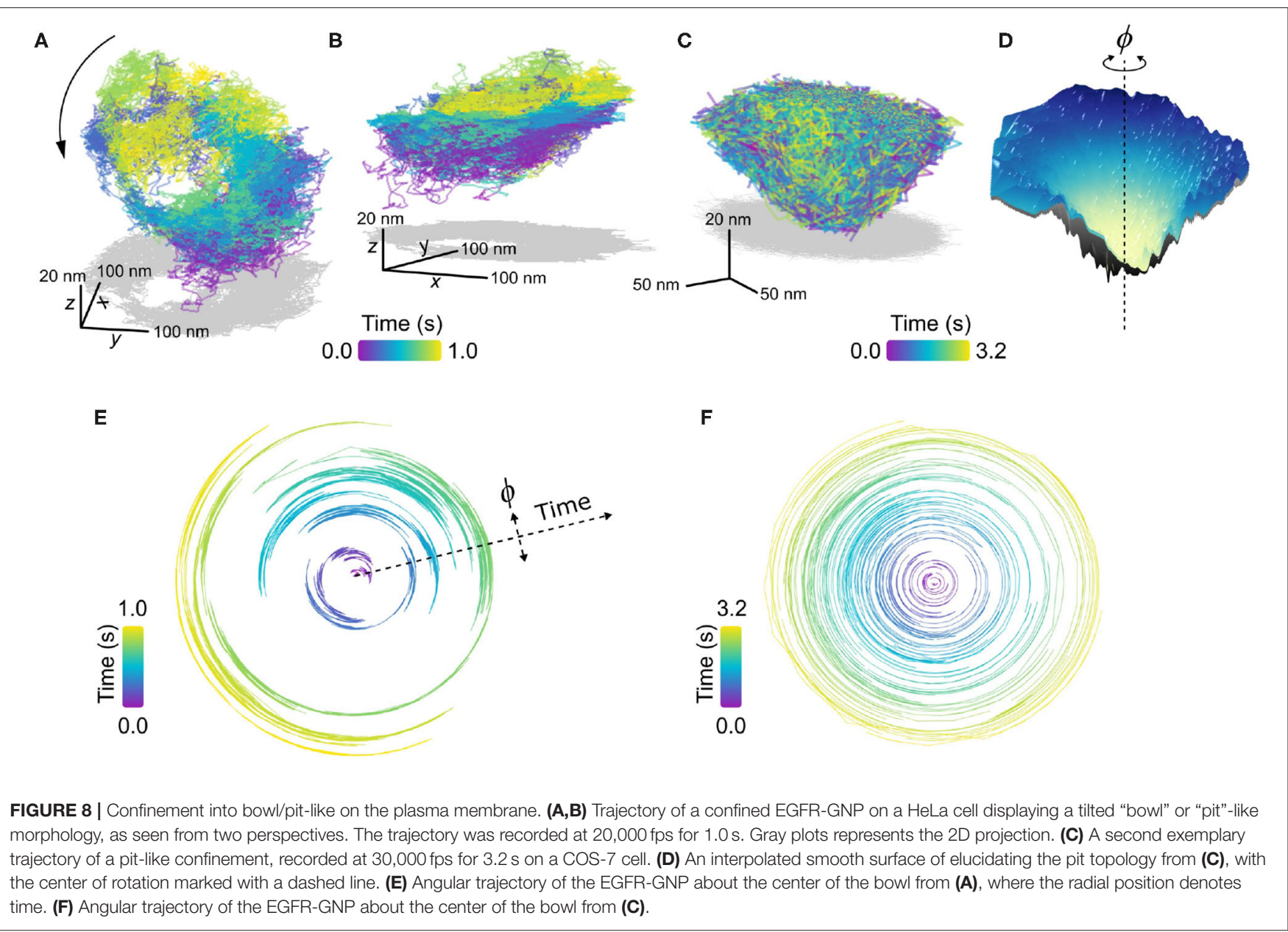

\subsection{Directed Motion}

A final example of another interesting mode of protein mobility is that of motion in a single concerted direction, as shown in Figure 9. In the cellular context, such directed motion is typically due to active transport processes mediated by motor proteins that move organelles and endocytosed vesicles along the intracellular network of cytoskeletal filaments (Caspi et al., 2000; Verhey et al., 2011; Granger et al., 2014). We might suppose in the examples shown in Figure 9 that EGFR-GNP is internalized within a vesicle inside of the cell, and is being trafficked within the cellular corpus.

In Figure 9A, we consider an example recorded at 10,000 fps wherein one sees a concerted diffusion along a given direction for several seconds that is then followed by a reverse walk and then a change in direction near orthogonal to the previous. Inspection of the trajectory in $3 \mathrm{D}$ reveals in fact that the second leg of the walk lies above the first, suggesting a switch in the track along which the walk occurs. The typical track width is seen to be around $20 \mathrm{~nm}$.

A trajectory with such a persistent directionality is described as being super-diffusive as the time-dependence of the MSD as $\alpha>1$. In Figure 9B, we present the macroscopic MSD computed by taking the complete trajectory at once, and find $\alpha=1.98$, which one can visualize through the upward curving slope of the MSD. The inset of Figure 9B presents the same MSD plotted on a log-log scale wherein on can see clearly the superdiffusive transport behavior manifest over longer time scales $(\tau>0.01 \mathrm{~s})$ and at shorter time scales $(\tau>0.01 \mathrm{~s})$ a more subdiffusive time-dependence is apparent. We can explore this subdiffusive aspect of the trajectory more closely by considering the rolling temporal exponent, shown in Figure 9C, for a temporal window of $T_{w}=100 \mathrm{~ms}$. Here, one sees the temporal exponent fluctuates around $\alpha \approx 0.54$. One can anticipate such 'confined'like behavior in the short-time diffusion since the EGFR-GNP is likely confined to a vesicle which itself is tethered to the intracellular filament. Over longer timescales the processive mobility begins to emerge, reflected in the increasing value in $\alpha$.

A second example, of super-diffusive mobility is presented in Figure 9D for a trajectory recorded at 1,000 fps over $30 \mathrm{~s}$ observation time. In this example, a strongly linear track with a mean width of $10 \mathrm{~nm}$ is evident. Careful analysis of such trajectories shown in Figures 9A,D allows us to not only identify the diffusive mode of transport but other important characteristics such as the velocity, step size and dwell time associated with the specific motor proteins which traffic cargo along these filaments within the cell. Taking into consideration that here we monitor the movement of EGFR most likely 
A

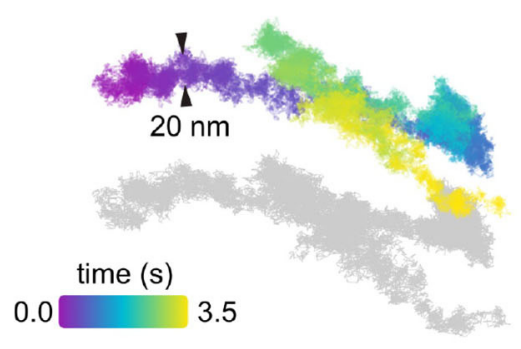

B

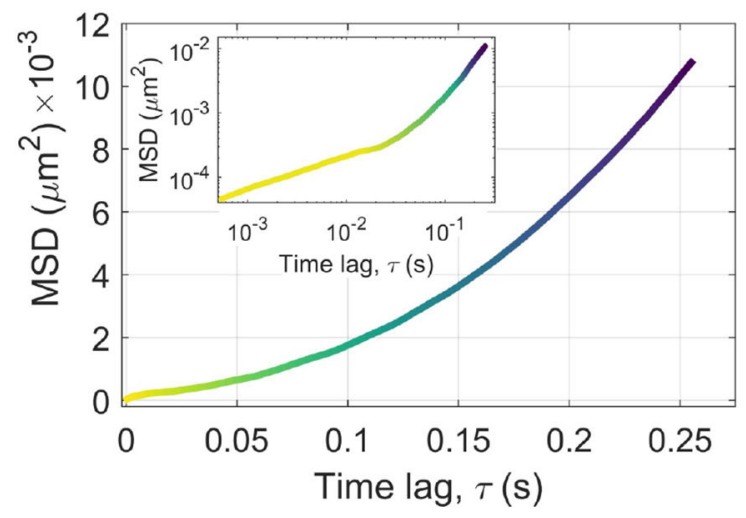

C

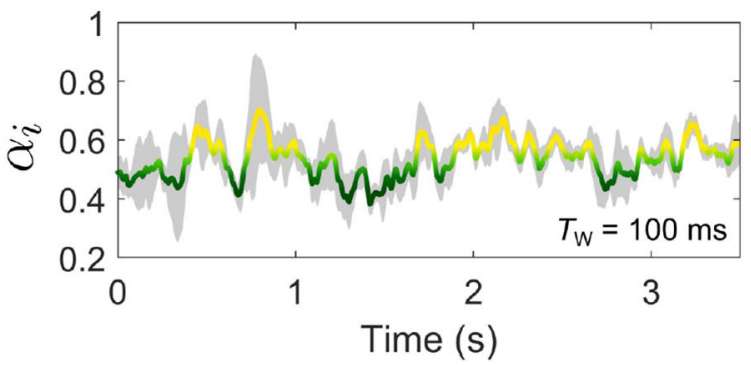

D

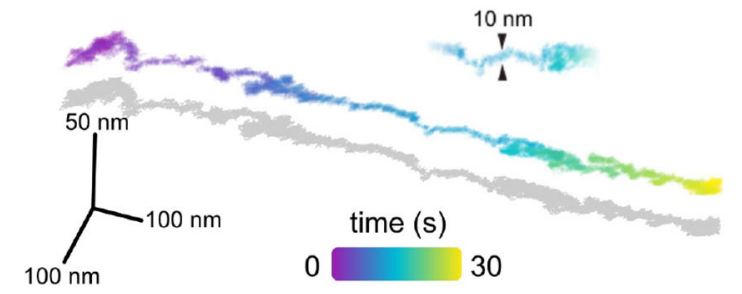

E

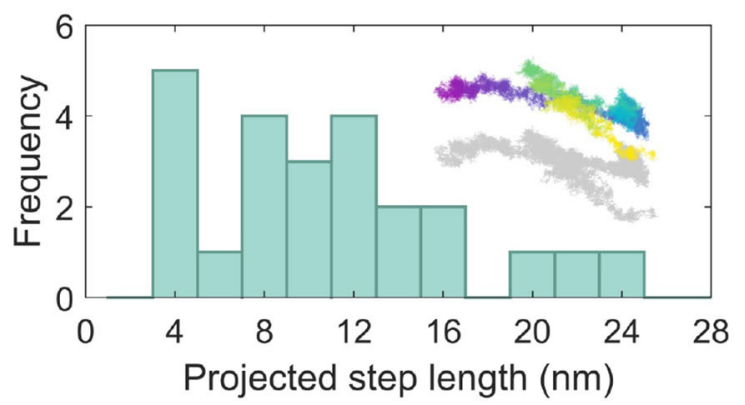

$\mathbf{F}$

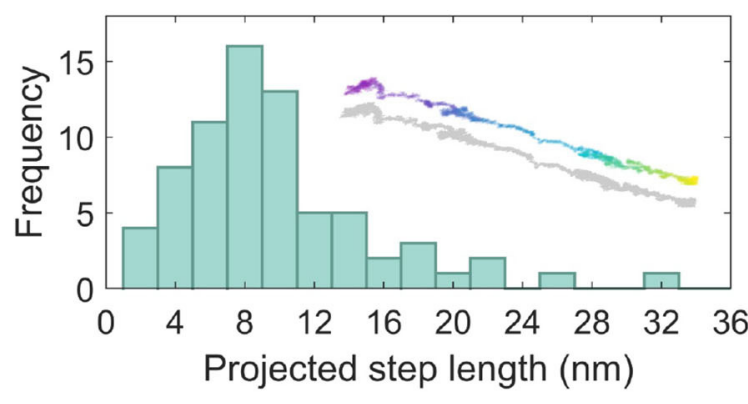

FIGURE 9 | Directed motion of the EGFR-GNP probe. (A) A trajectory recorded at 10,000 fps for 3.5 s showing a concerted linear trafficking of the EGFR-GNP probe on a HeLa cell. The typical width of the trajectory track is $20 \mathrm{~nm}$. (B) The MSD of the trajectory from (A), showing super-diffusive mobility. The inset is the same MSD plotted on a log-log scale. (C) The temporal exponent of the trajectory from (A) for a rolling window of $T_{w}=100 \mathrm{~ms}$. (D) A second example of a 3D trajectory showing directed motion, recorded at $1,000 \mathrm{fps}$ for a duration of $30 \mathrm{~s}$. The width of the track is approximately $10 \mathrm{~nm}$. (E) A histogram of prominent steps projected into the direction of travel, from the trajectory in (A). (F) A histogram of prominent steps projected onto the direction of travel, taken the trajectory shown in (D).

trapped in an intracellular vesicle, the inspection of these features of in vivo transport is fraught with complications and nuanced interpretation. In addition to active transport, velocity and step size will be affected by indirect movements such as polymerization or depolymerization of the filament it is attached to. Moreover, one must be mindful of the region within the cell where the EGFR-GNP is being tracked. If the filament is not lying flat to the coverslip, for example being located near the nucleus and orientated randomly, then what one observes in $2 \mathrm{D}$ is a projection of the true trajectory and hence it is not an accurate representation of the true step size. In iSCAT one can capture the $3 \mathrm{D}$ position so such a problem can be remedied. However, since the trajectories in Figure 9 appear near-parallel to the focal plane, one can assume this skew is not significant in these recordings. Whilst it is beyond the scope of this work to draw specific conclusions as to the behavior exhibited in Figure 9, it is nonetheless interesting to inspect what iSPT can provide in exploring in vivo transport and the issues surrounding it.

To inspect the occurrence of a preferred step length in the trajectories of Figures 9A,D we define a linear axis that lies through the global direction of travel and onto this we project each individual step. We then may compute the accumulated distance walked by summing all projected steps and by using a peak-finding algorithm we can extract positions separating prominent steps along the walk. Figure 9E presents a histogram of the prominent projected steps from the trajectory shown in Figure 9A (denoted by the inset) wherein one sees a preference for steps with an approximate integer multiple of $4 \mathrm{~nm}$, most of which in a range up to $24 \mathrm{~nm}$. 
In Figure 9F, a similar step-size histogram is given for the trajectory of Figure 9D. In this latter example one also sees a similar distribution, but now with a stronger bias toward steps of $\approx 8 \mathrm{~nm}$.

Elucidation of the step size associated with specific motor proteins has been the subject of numerous in vitro experiments wherein individual filaments and single motor proteins are investigated under pristine conditions (e.g., Hirakawa et al., 2000; Köhler et al., 2003; Leduc et al., 2007; Ali et al., 2011; Hundt et al., 2016). Whilst much of these investigations to quantify the step size have been done with fluorescence microscopies, recent efforts have harnessed the improved resolution of iSPT to identify the exact stepping mechanism of single myosin V (Ortega Arroyo et al., 2014; Andrecka et al., 2015), also in vitro, which has remained a long standing matter of debate. The tracking and interpretation of transport mediated by motor proteins in vivo within the living cell is a much more complex scenario, since it can involve more than just one transport mechanism. Various studies have, for example, revealed a switch of the transporting motor protein species such as from myosin, which travels on actin filaments to kinesin, which travels upon microtubules (Provance et al., 2008; Kapitein et al., 2013). Additionally, these motor proteins not only alternate when transporting cargo, but can also be attached to the vesicle at the same time, requiring cooperative and even coordinated interactions (Gross et al., 2002; Levi et al., 2006; Ali et al., 2011). Furthermore, sub-division of the anticipated characteristic steps sizes can occur, as observed in in vitro experiments with kinesin (Leduc et al., 2007) and in live cells with myosin II (Fujita et al., 2019).

The step sizes and track widths of the trajectories in Figure 9 are in the range of step sizes reported for common molecular motor proteins including kinesin, myosin II, myosin $\mathrm{V}$, and VI in analogous in vitro and live cell measurements (Yildiz et al., 2003; Ökten et al., 2004; Leduc et al., 2007; Pierobon et al., 2009; Ali et al., 2011; Fujita et al., 2019) and in good agreement with the widths of $7 \mathrm{~nm}$ of single actin filaments and $25 \mathrm{~nm}$ of microtubules (Cooper, 2000). Therefore, these trajectories likely represent examples of active transport within the cell. However, lacking further information one may only speculate as to the molecular origin of these observed step sizes since the step size alone is not a distinctive characteristic of a particular motor protein and may be affected by interactions, cooperative and antagonistic effects in a living cell. One also has to consider that most of the in vivo and in vitro studies measure step size by labeling the motor protein itself, while we, in contrast, see the stepping behavior of the transported cargo.

To address the issue of intracellular transport more conclusively, one requires further investigation with a larger sample size to draw a more robust statistical analysis as well as measurement which harnesses fluorescence-based labeling to identify and visualize intracellular filaments and motor proteins in parallel with the iSPT tracking. Nonetheless, these initial efforts and recent similar in vivo iSPT tracking of cytoplasmatic vesicle transport (Huang et al., 2017b) demonstrate that iSPT is well-suited to investigate the nanoscopic minutia of intracellular transport events in living cells.

\section{DISCUSSION: ISPT FOR INVESTIGATION OF MOBILITY WITHIN THE LIVE CELL}

\section{iSPT Microscopy}

iSPT microscopy over the past few years has demonstrated great potential for new avenues of investigation into membrane diffusion, whether on synthetic or live cell membranes, owing to its high detection sensitivity affording fast and precise particle tracking. The power of the technique lies in the exploitation of interference between the light scattered by the probe and that which naturally reflects off the sample coverslip. For this reason iSPT, and more generally iSCAT microscopy, does not require any special equipment, save for a suitably fast camera to achieve high imaging rates. This makes iSCAT microscopy easy to implement on existing microscopes, including commercial fluorescence microscopes, and as both microscopies are mutually compatible (Kukura et al., 2009) they can also be performed in parallel. This is particularly important in live cell imaging where fluorescent labeling is useful for identifying specific cell and membrane components and features.

\section{Phototoxicity}

One important issue in live cell imaging is the role of photodamage associated with high laser intensities commonly associated with high-resolution microscopies (Laissue et al., 2017). The threshold for potential cell damage depends on the particular cells in use as well as the particular aspect of the membrane and cell biology under investigation (Wäldchen et al., 2015). We have demonstrated previously one can achieve high framerate and accurate tracking of EGFR-GNP probes on HeLa cells using illumination powers that are tolerable for cell viability (Taylor et al., 2019), being on the order of no more than $10 \mathrm{kWcm}^{-2}$. In general, using illumination wavelengths toward the red end of the spectrum (beginning around $600 \mathrm{~nm}$ ) appears to be the most compatible for preserving cell viability. One asset of iSCAT microscopy is that, unlike fluorescence, one has complete flexibility in choice of the wavelength used and thus it is possible to perform iSCAT microscopies at wavelengths that preserve cell health whilst also maintaining high framerate imaging and good signal-to-noise ratio.

\section{Influence of the Probe}

An issue often raised in SPT is to what extent the probe influences the diffusive process and membrane biology under investigation. The label, depending on its geometry and functionalization introduces multivalent binding, non-specific binding as well as the physical size adding steric hindrances to the crowded membrane environment. The latter of which is an important consideration for colloidal probes particularly used for iSPT. In addition, because the localization precision now can approach the few-nm length scale, the influence of the GNP-to-ligand linker becomes of interest, in particular, its length and flexibilty, and how these might affect the accuracy in localization of the associated protein. Many of these questions are not fully answered, but recent studies have investigated these issues of multi-valent labeling and efforts to control it (Reina et al., 2018; Liao et al., 2019), as well as the influence of colloidal probe size 
(Ritchie et al., 2005; Clausen and Lagerholm, 2011; Etoc et al., 2018). In the case of the latter, the size of the probe may not have significant effects on diffusion within the cell as one would have initially assumed.

Similarly, in previous work, we also found the use of $48 \mathrm{~nm}$ EGF-GNP probes seemingly did not impair the EGFR signaling pathway or uptake via clathrin-mediated endocytosis (Taylor et al., 2019). To fully address this matter, one must systematically explore membrane diffusion for colloidal probes of differing sizes, as it has been recently demonstrated for synthetic membranes (Liao et al., 2019). Usage of smaller colloidal probes on the live cell membrane is challenging solely due to the dynamic speckle background which presents the biggest hurdle to overcome. However, new methods for background modeling and subtraction in interferometric microscopies are constantly being proposed (Cheng and Hsieh, 2017; Taylor et al., 2019; Lin et al., 2020; Mahmoodabadi et al., 2020), and so it is realistic that the challenge posed by the cell background will be met within the near future.

\section{CONCLUSION AND OUTLOOK}

iSPT microscopy stands as a promising technique to expand the frontiers of investigation into single molecule diffusion and membrane organization. The high resolution visualization of protein mobility in $3 \mathrm{D}$ provides a wealth of information hitherto inaccessible through conventional fluorescence microscopies. When coupled with complementary techniques such as superresolution fluorescence and correlative electron microscopies, one is presented with an impressive tool box that could help addressing long standing questions regarding dynamic membrane organization such as the size, lifetime, and diffusive properties of membrane raft domains. In this work we have focused on demonstrating the applicability of high-resolution iSPT microscopy on the live cell, and as such rather than providing an exhaustive study into the membrane biology of the receptor. We remark that each of the phenomena discussed herein warrants an extended investigation of its own to fully characterize and explain the behaviors observed.

Furthermore, more broadly, iSCAT microscopy is an emergent and rapidly growing technique that is finding increasing applications in nanoscale biology, with aims ranging from label-free detection and mass-determination of single proteins and complexes, to the detection of viruses and vesicles as well as live cell imaging. Recent interdisciplinary efforts to model the iPSF as well as to address dynamic speckle background through machine learning opens new paths to exciting live-cell applications. This will allow the use of smaller scattering probes, multiple distinct scattering labels for co-labeling investigations as well as being able to perform imaging and iSPT over extended axial ranges within deeply scattering tissues.

\section{MATERIALS AND METHODS}

\section{Widefield iSCAT Microscopy}

Laser light from a supercontinuum white-light laser (NKT Photonics) was filtered down to $\lambda_{\text {iSCAT }}=550 \pm 15 \mathrm{~nm}$ through a
Varia filter box. An 100x oil-immersion (NA = 1.4) objective was used to give a field of view of $5 \times 5 \mu \mathrm{m}^{2}$, which was then imaged onto $128 \times 128$ pixels of a high-speed camera (Vision Research, Phantom, Miro LAB 3a10).

In practice, illumination can be from any coherent light source, for gold nanoparticles green light is optimal. The illumination is unpolarized to minimize polarization artifacts which can arise for high-NA imaging. The objective is oilimmersion to maximize collection efficiency.

\section{Confocal Fluorescence Imaging}

A Zeiss LSM 800 was used with a water-dipping objective (40x), and modified to accommodate an iSCAT microscope underneath. A wavelength of $650 \mathrm{~nm}$ was chosen to avoid overlapping with the wide-field iSCAT imaging wavelength and to mitigate potential damage to live cells.

\section{Cell Culturing and Fluorescent Labeling}

HeLa and COS-7 cells were grown in DMEM (Gibco Invitrogen) supplemented with $10 \%$ fetal calf serum (FCS, Life Technologies) in a humidified atmosphere at $37^{\circ} \mathrm{C}$ and $5 \% \quad \mathrm{CO}_{2}$. For measurement, HeLa cells were plated onto a glass-bottomed sample dish (Ibidi $\mathrm{GmBH}$ ) and grown to 70\% confluency. Before measurement, each dish was rinsed twice with warmed PBS-BSA solution, serum starved for several hours and rinsed again with warmed PBS-BSA. Imaging was done in Leibovitz's L-15 Medium (1.5 ml Gibco, Invitrogen).

For tracking over-expressed EGFR on COS-7 cells, cells were counted and 90,000 cells per dish were seeded. The next day, medium was replaced by $3 \mathrm{ml}$ fresh DMEM and the Lipofectamine 3000 transfection mix was added (prepared according to the manufacturer's protocol, ThermoFisher). [Details: $4.6 \mu \mathrm{l}$ Lipofectamine 3000, $2 \mu \mathrm{g}$ plasmid DNA (EGFREGFP, addgene \#32751) and $4 \mu \mathrm{l}$ P3000 Reagent]. For imaging, cells were rinsed twice with DPBS and $3 \mathrm{ml}$ of pre-warmed Leibovitz's L-15 medium (without phenol red, ThermoFisher) were added.

When placed on the microscope, each culture was held at $37^{\circ} \mathrm{C}$ by a home-built objective heater. A micropipette (Piezo Drill Tip, Eppendorf) was used to deliver $10 \mu \mathrm{l}$ of the EGFGNPs to a local region of the culture, with observation beginning immediately thereafter.

\section{Gold Nanoparticle Probes}

GNPs with a diameter of $48 \mathrm{~nm}$ were functionalized with monoclonal anti-biotin (British Biocell International), and GNPs with diameters of $20 \mathrm{~nm}$, functionalized with streptavidin (British Biocell International), were conjugated at a molar ratio of $1: 1$ with biotinylated EGF (ThermoFisher) at a concentration of $0.66 \mathrm{nM}$. PBS was used as a buffer. The solution containing EGF-GNP was agitated for several hours at $37^{\circ} \mathrm{C}$ to assist conjugation, purified through centrifugation and diluted up to a final concentration of $0.1 \mathrm{nM}$. Cationic GNPs with diameter $50 \mathrm{~nm}$ were purchased from Nanopartz Inc. (item CC11-50-POS-DIH-50-1) and diluted to a ratio 1:100 in water. 


\section{DATA AVAILABILITY STATEMENT}

The raw data supporting the conclusions of this article will be made available by the authors, without undue reservation.

\section{AUTHOR CONTRIBUTIONS}

RT and $\mathrm{CH}$ made iSCAT and fluorescence measurements. RT performed data analysis. $\mathrm{CH}$ prepared the live cell materials. RM and HD developed background correction routines for the iSCAT images. MK developed the combination of confocal fluorescence and iSCAT microscopy. VZ supervised interpretation and analysis of the data. AS supervised biological preparation and data interpretation. VS conceived and supervised the project. RT, $\mathrm{CH}, \mathrm{VZ}$, and AS wrote the manuscript. All authors discussed the results and commented on the manuscript.

\section{FUNDING}

This project was funded by an Alexander von Humboldt professorship, the Max Planck Society and the Research and

\section{REFERENCES}

Abulrob, A., Lu, Z., Baumann, E., Vobornik, D., Taylor, R., Stanimirovic, D., et al. (2010). Nanoscale imaging of epidermal growth factor receptor clustering: effects of inhibitors. J. Biol. Chem. 285, 3145-3156. doi: 10.1074/jbc.M109.073338

Albrecht, D., Winterflood, C. M., and Ewers, H. (2015). Dual color single particle tracking via nanobodies. Methods Appl. Fluoresc. 3:024001. doi: 10.1088/2050-6120/3/2/024001

Alcor, D., Gouzer, G., and Triller, A. (2009). Single-particle tracking methods for the study of membrane receptors dynamics. Eur. J. Neurosci. 30, 987-997. doi: 10.1111/j.1460-9568.2009.06927.x

Ali, M. Y., Kennedy, G. G., Safer, D., Trybus, K. M., Sweeney, H. L., and Warshaw, D. M. (2011). Myosin Va and Myosin VI coordinate their steps while engaged in an in vitro tug of war during cargo transport. Proc. Natl. Acad. Sci. U.S.A. 108, E535-E541. doi: 10.1073/pnas.1104298108

Andrecka, J., Arroyo, J. O., Takagi, Y., de Wit, G., Fineberg, A., MacKinnon, L., et al. (2015). Structural dynamics of myosin 5 during processive motion revealed by interferometric scattering microscopy. eLife 4:e05413. doi: 10.7554/eLife.05413

Arteaga, C. L., and Engelman, J. A. (2014). ErbB receptors: from oncogene discovery to basic science to mechanism-based cancer therapeutics. Cancer Cell 25, 282-303. doi: 10.1016/j.ccr.2014.02.025

Bag, N., Huang, S., and Wohland, T. (2015). Plasma membrane organization of epidermal growth factor receptor in resting and ligand-bound states. Biophys. J. 109, 1925-1936. doi: 10.1016/j.bpj.2015.09.007

Bouchaud, J.-P., and Georges, A. (1990). Anomalous diffusion in disordered media: statistical mechanisms, models and physical applications. Phys. Rep. 195, 127-293. doi: 10.1016/0370-1573(90)90099-N

Caspi, A., Granek, R., and Elbaum, M. (2000). Enhanced diffusion in active intracellular transport. Phys. Rev. Lett. 85, 5655-5658. doi: 10.1103/PhysRevLett.85.5655

Cheng, C.-Y., and Hsieh, C.-L. (2017). Background estimation and correction for high-precision localization microscopy. ACS Photon. 4, 1730-1739. doi: 10.1021/acsphotonics.7b00238

Chung, I., Akita, R., Vandlen, R., Toomre, D., Schlessinger, J., and Mellman, I. (2010). Spatial control of egf receptor activation by reversible dimerization on living cells. Nature 464, 783-787. doi: 10.1038/nature08827
Training Grant 1962 (Dynamic Interactions at Biological Membranes) of the German Research Foundation. RT acknowledges an Alexander von Humboldt fellowship. AS was also supported by grants from the German Research Foundation (grant nos. SCHA965/6-2 and SCHA965/9-1).

\section{ACKNOWLEDGMENTS}

The authors wish to thank Jennifer Lühr for assisting with the development of GNP pipetting, Moritz Grob, and David Albrecht for careful reading of the manuscript and insightful comments, Simone Ihloff for assistance with the culturing of cells, Navid Bonakdar, Maksim Schwab, Oliver Bittel, and Lothar Meier for designing and fabricating the sample heating system.

\section{SUPPLEMENTARY MATERIAL}

The Supplementary Material for this article can be found online at: https://www.frontiersin.org/articles/10.3389/fcell.2020. 590158/full\#supplementary-material

Clausen, M., and Lagerholm, C. (2011). The probe rules in single particle tracking. Curr. Protein Pept. Sci. 12, 699-713. doi: 10.2174/138920311798841672

Clausen, M. P., and Lagerholm, B. C. (2013). Visualization of plasma membrane compartmentalization by high-speed quantum dot tracking. Nano Lett. 13, 2332-2337. doi: 10.1021/nl303151f

Cooper, G. M. (2000). The Cell: A Molecular Approach, 2nd Edn. Structure and Organization of Actin Filaments. Sunderland, MA: Sinauer Associates (2000). Available online at: https:/www.ncbi.nlm.nih.gov/books/NBK9908/

De Brabander, M., Geuens, G., Nuydens, R., Moeremans, M., and De Mey, J. (1986). Microtubule-dependent intracellular motility investigated with nanometer particle video ultramicroscopy (nanovid ultramicroscopy). Ann. N. Y. Acad. Sci. 466, 666-668. doi: 10.1111/j.1749-6632.1986.tb38448.x

De Brabander, M., Nuydens, R., Geerts, H., and Hopkins, C. (1988). Dynamic behavior of the transferrin receptor followed in living epidermoid carcinoma (a431) cells with nanovid microscopy. Cell Motil. Cytoskel. 9, 30-47. doi: 10.1002/cm.970090105

de Bruin, K., Ruthardt, N., Von Gersdorff, K., Bausinger, R., Wagner, E., Ogris, M., et al. (2007). Cellular dynamics of EGF receptor-targeted synthetic viruses. Mol. Ther. 15, 1297-1305. doi: 10.1038/sj.mt.6300176

de Wit, G., Albrecht, D., Ewers, H., and Kukura, P. (2018). Revealing compartmentalized diffusion in living cells with interferometric scattering microscopy. Biophys. J. 114, 2945-2950. doi: 10.1016/j.bpj.2018.05.007

Den Hartigh, J. C., van Bergen en Henegouwen, P. M. P., Verkleij, A. J., and Boonstra, J. (1992). The EGF receptor is an actin-binding protein. J. Cell Biol. 119, 349-355. doi: 10.1083/jcb.119.2.349

Deschout, H., Zanacchi, F. C., Mlodzianoski, M., Diaspro, A., Bewersdorf, J., Hess, S. T., et al. (2014). Precisely and accurately localizing single emitters in fluorescence microscopy. Nat. Methods 11:253. doi: 10.1038/ nmeth. 2843

Destainville, N., Dumas, F., and Salomé, L. (2008). What do diffusion measurements tell us about membrane compartmentalisation? Emergence of the role of interprotein interactions. J. Chem. Biol. 1, 37-48. doi: 10.1007/s12154-008-0005-3

Dix, J. A., and Verkman, A. S. (2008). Crowding effects on diffusion in solutions and cells. Annu. Rev. Biophys. 37, 247-263. doi: 10.1146/annurev.biophys.37.032807.125824

Erazo-Oliveras, A., Fuentes, N. R., Wright, R. C., and Chapkin, R. S. (2018). Functional link between plasma membrane spatiotemporal dynamics, cancer 
biology, and dietary membrane-altering agents. Cancer Metastasis. Rev. 37, 519-544. doi: 10.1007/s10555-018-9733-1

Etoc, F., Balloul, E., Vicario, C., Normanno, D., Liße, D., Sittner, A., et al. (2018). Non-specific interactions govern cytosolic diffusion of nanosized objects in mammalian cells. Nat. Mater. 17, 740-746. doi: 10.1038/s41563-018-0120-7

Freed, D. M., Bessman, N. J., Kiyatkin, A., Salazar-Cavazos, E., Byrne, P. O., Moore, J. O., et al. (2017). EGFR ligands differentially stabilize receptor dimers to specify signaling kinetics. Cell 171, 683-695. doi: 10.1016/j.cell.2017.09.017

Fujita, K., Ohmachi, M., Ikezaki, K., Yanagida, T., and Iwaki, M. (2019). Direct visualization of human myosin ii force generation using DNA origami-based thick filaments. Commun. Biol. 2, 1-11. doi: 10.1038/s42003-019-0683-0

Fujiwara, T., Ritchie, K., Murakoshi, H., Jacobson, K., and Kusumi, A. (2002). Phospholipids undergo hop diffusion in compartmentalized cell membrane. J. Cell Biol. 157, 1071-1082. doi: 10.1083/jcb.200202050

Fujiwara, T. K., Iwasawa, K., Kalay, Z., Tsunoyama, T. A., Watanabe, Y., Umemura, Y. M., et al. (2016). Confined diffusion of transmembrane proteins and lipids induced by the same actin meshwork lining the plasma membrane. Mol. Biol. Cell 27, 1101-1119. doi: 10.1091/mbc.E15-04-0186

Geerts, H., De Brabander, M., Nuydens, R., Geuens, S., Moeremans, M., De Mey, J., et al. (1987). Nanovid tracking: a new automatic method for the study of mobility in living cells based on colloidal gold and video microscopy. Biophys. J. 52, 775-782. doi: 10.1016/S0006-3495(87)83271-X

Gowrishankar, K., Ghosh, S., Saha, S., Rumamol, C., Mayor, S., and Rao, M. (2012). Active remodeling of cortical actin regulates spatiotemporal organization of cell surface molecules. Cell 149, 1353-1367. doi: 10.1016/j.cell.2012.05.008

Granger, E., McNee, G., Allan, V., and Woodman, P. (2014). The role of the cytoskeleton and molecular motors in endosomal dynamics. Semin. Dev. Cell Biol. 31, 20-29. doi: 10.1016/j.semcdb.2014.04.011

Gross, S. P., Tuma, M. C., Deacon, S. W., Serpinskays, A. S., Reilein, A. R., and Gelfand, V. I. (2002). Interactions and regulation of molecular motors in xenopus melanophores. J. Cell Biol. 156, 855-865. doi: 10.1083/jcb.200105055

Hirakawa, E., Higuchi, H., and Toyoshima, Y. Y. (2000). Processive movement of single 22s dynein molecules occurs only at low ATP concentrations. Proc. Natl. Acad. Sci. U.S.A. 97, 2533-2537. doi: 10.1073/pnas.050585297

Huang, Y.-F., Zhuo, G.-Y., Chou, C.-Y., Lin, C.-H., Chang, W., and Hsieh, C.-L. (2017a). Coherent brightfield microscopy provides the spatiotemporal resolution to study early stage viral infection in live cells. ACS Nano 11, 2575-2585. doi: 10.1021/acsnano.6b05601

Huang, Y.-F., Zhuo, G.-Y., Chou, C.-Y., Lin, C.-H., and Hsieh, C.-L. (2017b). Label-free, ultrahigh-speed, 3D observation of bidirectional and correlated intracellular cargo transport by coherent brightfield microscopy. Nanoscale 9 , 6567-6574. doi: 10.1039/C7NR00604G

Hundt, N., Steffen, W., Pathan-Chhatbar, S., Taft, M. H., and Manstein, D. J. (2016). Load-dependent modulation of non-muscle myosin-2a function by tropomyosin 4.2. Sci. Rep. 6:20554. doi: 10.1038/srep20554

Isichenko, M. B. (1992). Percolation, statistical topography, and transport in random media. Rev. Mod. Phys. 64:961. doi: 10.1103/RevModPhys.64.961

Jacobsen, V., Klotzsch, E., and Sandoghdar, V. (2007). "Chapter 9: Interferometric detection and tracking of nanoparticles," in Nano Biophotonics: Science and Technology, Vol. 3, eds H. Masuhara, S. Kawata, and F. Tokunaga (Amsterdam: Elsevier), 143-159. doi: 10.1016/S1574-0641(07)80014-3

Jacobson, K., Liu, P., and Lagerholm, B. C. (2019). The lateral organization and mobility of plasma membrane components. Cell 177, 806-819. doi: 10.1016/j.cell.2019.04.018

Jacobson, K., Mouritsen, O. G., and Anderson, R. G. (2007). Lipid rafts: at a crossroad between cell biology and physics. Nat. Cell Biol. 9, 7-14. doi: $10.1038 /$ ncb0107-7

Jaqaman, K., and Grinstein, S. (2012). Regulation from within: the cytoskeleton in transmembrane signaling. Trends Cell Biol. 22, 515-526. doi: 10.1016/j.tcb.2012.07.006

Jin, S., and Verkman, A. (2007). Single particle tracking of complex diffusion in membranes: simulation and detection of barrier, raft, and interaction phenomena. J. Phys. Chem. B 111, 3625-3632. doi: 10.1021/jp067187m

Kapitein, L. C., van Bergeijk, P., Lipka, J., Keijzer, N., Wulf, P. S., Katrukha, E. A., et al. (2013). Myosin- $\mathrm{V}$ opposes microtubule-based cargo transport and drives directional motility on cortical actin. Curr. Biol. 23, 828-834. doi: 10.1016/j.cub.2013.03.068
Kepten, E., Bronshtein, I., and Garini, Y. (2013). Improved estimation of anomalous diffusion exponents in single-particle tracking experiments. Phys. Rev. E 87:052713. doi: 10.1103/PhysRevE.87.052713

Kepten, E., Weron, A., Sikora, G., Burnecki, K., and Garini, Y. (2015). Guidelines for the fitting of anomalous diffusion mean square displacement graphs from single particle tracking experiments. PLOS ONE 10:e117722. doi: 10.1371/journal.pone.0117722

Köhler, D., Ruff, C., Meyhöfer, E., and Bähler, M. (2003). Different degrees of lever arm rotation control myosin step size. J. Cell Biol. 161, 237-241. doi: $10.1083 /$ jcb.200212039

Kovacs, E., Zorn, J. A., Huang, Y., Barros, T., and Kuriyan, J. (2015). A structural perspective on the regulation of the epidermal growth factor receptor. Annu. Rev. Biochem. 84, 739-764. doi: 10.1146/annurev-biochem-060614-034402

Krapf, D. (2018). Compartmentalization of the plasma membrane. Curr. Opin. Cell Biol. 53, 15-21. doi: 10.1016/j.ceb.2018.04.002

Kukura, P., Ewers, H., Müller, C., Renn, A., Helenius, A., and Sandoghdar, V. (2009). High-speed nanoscopic tracking of the position and orientation of a single virus. Nat. Methods 6, 923-927. doi: 10.1038/nmeth.1395

Kusumi, A., Fujiwara, T. K., Chadda, R., Xie, M., Tsunoyama, T. A., Kalay, Z., et al. (2012). Dynamic organizing principles of the plasma membrane that regulate signal transduction: commemorating the fortieth anniversary of singer and Nicolson's fluid-mosaic model. Annu. Rev. Cell Dev. Biol. 28, 215-250. doi: 10.1146/annurev-cellbio-100809-151736

Kusumi, A., Nakada, C., Ritchie, K., Murase, K., Suzuki, K., Murakoshi, H., et al. (2005). Paradigm shift of the plasma membrane concept from the two-dimensional continuum fluid to the partitioned fluid: high-speed singlemolecule tracking of membrane molecules. Annu. Rev. Biophys. Biomol. Struct. 34, 351-378. doi: 10.1146/annurev.biophys.34.040204.144637

Kusumi, A., Sako, Y., and Yamamoto, M. (1993). Confined lateral diffusion of membrane receptors as studied by single particle tracking (nanovid microscopy). Effects of calcium-induced differentiation in cultured epithelial cells. Biophys. J. 65, 2021-2040. doi: 10.1016/S0006-3495(93)81253-0

Kusumi, A., Tsunoyama, T. A., Hirosawa, K. M., Kasai, R. S., and Fujiwara, T. K. (2014). Tracking single molecules at work in living cells. Nat. Chem. Biol. 10, 524-532. doi: 10.1038/nchembio.1558

Laissue, P. P., Alghamdi, R. A., Tomancak, P., Reynaud, E. G., and Shroff, H. (2017). Assessing phototoxicity in live fluorescence imaging. Nat. Methods 14, 657-661. doi: 10.1038/nmeth.4344

Leduc, C., Ruhnow, F., Howard, J., and Diez, S. (2007). Detection of fractional steps in cargo movement by the collective operation of kinesin-1 motors. Proc. Natl. Acad. Sci. U.S.A. 104, 10847-10852. doi: 10.1073/pnas.0701864104

Levi, V., Serpinskaya, A. S., Gratton, E., and Gelfand, V. I. (2006). Organelle transport along microtubules in xenopus melanophores: evidence for cooperation between multiple motors. Biophys. J. 90, 318-327. doi: 10.1529/biophysj.105.067843

Liao, Y.-H., Lin, C.-H., Cheng, C.-Y., Wong, W. C., Juo, J.-Y., and Hsieh, C.L. (2019). Monovalent and oriented labeling of gold nanoprobes for the high-resolution tracking of a single-membrane molecule. ACS Nano 13, 10918-10928. doi: 10.1021/acsnano.9b01176

Lin, S., He, Y., Li, H., Zhang, P., and Chen, X.-W. (2020). Multiscale modeling and analysis of interferometric scattering microscopy. arXiv [Preprint]. Available online at: https://arxiv.org/abs/2004.10575 (accessed May 30, 2020).

Lin, Y.-H., Chang, W.-L., and Hsieh, C.-L. (2014). Shot-noise limited localization of single $20 \mathrm{~nm}$ gold particles with nanometer spatial precision within microseconds. Opt. Exp. 22, 9159-9170. doi: 10.1364/OE.22.009159

Lindfors, K., Kalkbrenner, T., Stoller, P., and Sandoghdar, V. (2004). Detection and spectroscopy of gold nanoparticles using supercontinuum white light confocal microscopy. Phys. Rev. Lett. 93:037401. doi: 10.1103/PhysRevLett.93. 037401

Linggi, B., and Carpenter, G. (2006). ErbB receptors: new insights on mechanisms and biology. Trends Cell Biol. 16, 649-656. doi: 10.1016/j.tcb.2006.10.008

Liu, Y.-L., Perillo, E. P., Liu, C., Yu, P., Chou, C.-K., Hung, M.-C., et al. (2016). Segmentation of $3 \mathrm{~d}$ trajectories acquired by tsunami microscope: an application to EGFR trafficking. Biophys. J. 111, 2214-2227. doi: 10.1016/j.bpj.2016.09.041

Lyman, E., Hsieh, C.-L., and Eggeling, C. (2018). From dynamics to membrane organization: experimental breakthroughs occasion a "modeling manifesto." Biophys. J. 115, 595-604. doi: 10.1016/j.bpj.2018.07.012 
Mahmoodabadi, R. G., Taylor, R. W., Kaller, M., Spindler, S., Mazaheri, M., Kasaian, K., et al. (2020). Point spread function in interferometric scattering microscopy (iSCAT). part i: aberrations in defocusing and axial localization. Opt. Exp. 28, 25969-25988. doi: 10.1364/OE.401374

Manzo, C., and Garcia-Parajo, M. F. (2015). A review of progress in single particle tracking: from methods to biophysical insights. Rep. Prog. Phys. 78:124601. doi: 10.1088/0034-4885/78/12/124601

Martin, D. S., Forstner, M. B., and Käs, J. A. (2002). Apparent subdiffusion inherent to single particle tracking. Biophys. J. 83, 2109-2117. doi: 10.1016/S0006-3495(02)73971-4

Mashaghi, A., Mashaghi, S., Reviakine, I., Heeren, R. M., Sandoghdar, V., and Bonn, M. (2014). Label-free characterization of biomembranes: from structure to dynamics. Chem. Soc. Rev. 43, 887-900. doi: 10.1039/C3CS60243E

Mazaheri, M., Ehrig, J., Shkarin, A., Zaburdaev, V., and Sandoghdar, V. (2020). Ultrahigh-speed imaging of rotational diffusion on a lipid bilayer. Nano Lett. 20, 7213-7219. doi: 10.1021/acs.nanolett.0c02516

McDonald, M. P., Gemeinhardt, A., König, K., Piliarik, M., Schaffer, S., Völkl, S., et al. (2018). Visualizing single-cell secretion dynamics with single-protein sensitivity. Nano Lett. 18, 513-519. doi: 10.1021/acs.nanolett. $7 \mathrm{~b} 04494$

Meroz, Y., and Sokolov, I. M. (2015). A toolbox for determining subdiffusive mechanisms. Phys. Rep. 573, 1-29. doi: 10.1016/j.physrep.2015. 01.002

Metzler, R., Jeon, J.-H., Cherstvy, A. G., and Barkai, E. (2014). Anomalous diffusion models and their properties: non-stationarity, non-ergodicity, and ageing at the centenary of single particle tracking. Phys. Chem. Chem. Phys. 16, 24128-24164. doi: 10.1039/C4CP03465A

Metzler, R., and Klafter, J. (2000). The random walk's guide to anomalous diffusion: a fractional dynamics approach. Phys. Rep. 339, 1-77. doi: 10.1016/S0370-1573(00)00070-3

Montroll, E. W., and Lebowitz, J. (1976). Studies in Statistical Mechanics. NorthHolland Publishing Company.

Munder, M. C., Midtvedt, D., Franzmann, T., Nuske, E., Otto, O., Herbig, M., et al. (2016). A pH-driven transition of the cytoplasm from a fluid-to a solid-like state promotes entry into dormancy. eLife 5:e09347. doi: 10.7554/eLife.09347

Oda, K., Matsuoka, Y., Funahashi, A., and Kitano, H. (2005). A comprehensive pathway map of epidermal growth factor receptor signaling. Mol. Syst. Biol. 1:2005.0010. doi: $10.1038 / \mathrm{msb} 4100014$

Ökten, Z., Churchman, L. S., Rock, R. S., and Spudich, J. A. (2004). Myosin VI walks hand-over-hand along actin. Nat. Struct. Mol. Biol. 11, 884-887. doi: $10.1038 / \mathrm{nsmb} 815$

Ortega Arroyo, J., Andrecka, J., Spillane, K., Billington, N., Takagi, Y., Sellers, J., et al. (2014). Label-free, all-optical detection, imaging, and tracking of a single protein. Nano Lett. 14, 2065-2070. doi: 10.1021/nl500234t

Parthasarathy, R. (2012). Rapid, accurate particle tracking by calculation of radial symmetry centers. Nat. Methods 9:724. doi: 10.1038/nmeth.2071

Pierobon, P., Achouri, S., Courty, S., Dunn, A. R., Spudich, J. A., Dahan, M., et al. (2009). Velocity, processivity, and individual steps of single myosin v molecules in live cells. Biophys. J. 96, 4268-4275. doi: 10.1016/j.bpj.2009.02.045

Pike, L. J. (2006). Rafts defined: a report on the keystone symposium on lipid rafts and cell function. J. Lipid Res. 47, 1597-1598. doi: 10.1194/jlr.E600002JLR200

Piliarik, M., and Sandoghdar, V. (2014). Direct optical sensing of single unlabelled proteins and super-resolution imaging of their binding sites. Nat. Commun. 5, 1-8. doi: 10.1038/ncomms5495

Pinaud, F., Clarke, S., Sittner, A., and Dahan, M. (2010). Probing cellular events, one quantum dot at a time. Nat. Methods 7, 275-285. doi: 10.1038/nmeth.1444

Provance, D. W., Addison, E. J., Wood, P. R., Chen, D. Z., Silan, C. M., and Mercer, J. A. (2008). Myosin-VB functions as a dynamic tether for peripheral endocytic compartments during transferrin trafficking. BMC Cell Biol. 9:44. doi: 10.1186/1471-2121-9-44

Reina, F., Galiani, S., Shrestha, D., Sezgin, E., de Wit, G., Cole, D., et al. (2018). Complementary studies of lipid membrane dynamics using iSCAT and super-resolved fluorescence correlation spectroscopy. J. Phys. D Appl. Phys. 51:235401. doi: 10.1088/1361-6463/aac04f

Renner, M., Wang, L., Levi, S., Hennekinne, L., and Triller, A. (2017). A simple and powerful analysis of lateral subdiffusion using single particle tracking. Biophys. J. 113, 2452-2463. doi: 10.1016/j.bpj.2017.09.017
Ritchie, K., Shan, X.-Y., Kondo, J., Iwasawa, K., Fujiwara, T., and Kusumi, A. (2005). Detection of non-brownian diffusion in the cell membrane in single molecule tracking. Biophys. J. 88, 2266-2277. doi: 10.1529/biophysj.104. 054106

Roskoski, R. Jr. (2014). The ErBb/HER family of protein-tyrosine kinases and cancer. Pharmacol. Res. 79, 34-74. doi: 10.1016/j.phrs.2013. 11.002

Sako, Y., Minoghchi, S., and Yanagida, T. (2000). Single-molecule imaging of egfr signalling on the surface of living cells. Nat. Cell Biol. 2, 168-172. doi: $10.1038 / 35004044$

Saxton, M. J. (1994). Anomalous diffusion due to obstacles: a Monte Carlo study. Biophys. J. 66, 394-401. doi: 10.1016/S0006-3495(94)80789-1

Saxton, M. J. (1996). Anomalous diffusion due to binding: a Monte Carlo study. Biophys. J. 70, 1250-1262. doi: 10.1016/S0006-3495(96)79682-0

Saxton, M. J. (2012). Wanted: a positive control for anomalous subdiffusion. Biophys. J. 103, 2411-2422. doi: 10.1016/j.bpj.2012.10.038

Saxton, M. J., and Jacobson, K. (1997). Single-particle tracking: applications to membrane dynamics. Annu. Rev. Biophys. 26, 373-399. doi: 10.1146/annurev.biophys.26.1.373

Sezgin, E., Levental, I., Mayor, S., and Eggeling, C. (2017). The mystery of membrane organization: composition, regulation and roles of lipid rafts. Nat. Rev. Mol. 18:361. doi: 10.1038/nrm.2017.16

Shen, H., Tauzin, L. J., Baiyasi, R., Wang, W., Moringo, N., Shuang, B., et al. (2017). Single particle tracking: from theory to biophysical applications. Chem. Rev. 117, 7331-7376. doi: 10.1021/acs.chemrev.6b00815

Sorkin, A., and Goh, L. K. (2009). Endocytosis and intracellular trafficking of ErbBs. Exp. Cell Res. 315, 683-696. doi: 10.1016/j.yexcr.2008. 07.029

Spindler, S., Ehrig, J., König, K., Nowak, T., Piliarik, M., Stein, H. E., et al. (2016). Visualization of lipids and proteins at high spatial and temporal resolution via interferometric scattering (iSCAT) microscopy. J. Phys. D Appl. Phys. 49:274002. doi: 10.1088/0022-3727/49/27/274002

Taylor, R. W., Mahmoodabadi, R. G., Rauschenberger, V., Giessl, A., Schambony, A., and Sandoghdar, V. (2019). Interferometric scattering microscopy reveals microsecond nanoscopic protein motion on a live cell membrane. Nat. Photon. 13, 480-487. doi: 10.1038/s41566-019-0414-6

Taylor, R. W., and Sandoghdar, V. (2019). "Interferometric scattering (iSCAT) microscopy and related techniques," in Label-Free Super-Resolution Microscopy ed V. Astratov (Cham:Springer), 25-65. doi: 10.1007/978-3-030-21722-8_2

Thompson, R. E., Larson, D. R., and Webb, W. W. (2002). Precise nanometer localization analysis for individual fluorescent probes. Biophys. J. 82, 2775-2783. doi: 10.1016/S0006-3495(02)75618-X

Tomas, A., Futter, C. E., and Eden, E. R. (2014). EGF receptor trafficking: consequences for signaling and cancer. Trends Cell. Biol. 24, 26-34. doi: $10.1016 /$ j.tcb.2013.11.002

Verhey, K. J., Kaul, N., and Soppina, V. (2011). Kinesin assembly and movement in cells. Annu. Rev. Biophys. 40, 267-288. doi: 10.1146/annurev-biophys-042910-155310

Vestergaard, C. L. (2016). Optimizing experimental parameters for tracking of diffusing particles. Phys. Rev. E 94:022401. doi: 10.1103/PhysRevE.94 022401

Wäldchen, S., Lehmann, J., Klein, T., Van De Linde, S., and Sauer, M. (2015). Lightinduced cell damage in live-cell super-resolution microscopy. Sci. Rep. 5:15348 doi: $10.1038 /$ srep 15348

Weber, S. C., Thompson, M. A., Moerner, W. E., Spakowitz, A. J., and Theriot, J. A. (2012). Analytical tools to distinguish the effects of localization error, confinement, and medium elasticity on the velocity autocorrelation function. Biophys. J. 102, 2443-2450. doi: 10.1016/j.bpj.2012.03.062

Weeks, E. R., and Weitz, D. (2002). Properties of cage rearrangements observed near the colloidal glass transition. Phys. Rev. Lett. 89:095704. doi: 10.1103/PhysRevLett.89.095704

Wieser, S., and Schütz, G. J. (2008). Tracking single molecules in the live cell plasma membrane-do's and don't's. Methods 46, 131-140. doi: 10.1016/j.ymeth.2008.06.010

Woringer, M., Izeddin, I., Favard, C., and Berry, H. (2020). Anomalous subdiffusion in living cells: bridging the gap between experiments and realistic models through collaborative challenges. Front. Phys. 8:134. doi: $10.3389 /$ fphy. 2020.00134 
Wu, H.-M., Lin, Y.-H., Yen, T.-C., and Hsieh, C.-L. (2016). Nanoscopic substructures of raft-mimetic liquid-ordered membrane domains revealed by high-speed single-particle tracking. Sci. Rep. 6:20542. doi: 10.1038/srep 20542

Ye, Z., Wang, X., and Xiao, L. (2019). Single-particle tracking with scattering-based optical microscopy. Anal. Chem. 91, 15327-15334. doi: 10.1021 /acs.analchem.9b02760

Yildiz, A., Forkey, J. N., McKinney, S. A., Ha, T., Goldman, Y. E., and Selvin, P. R. (2003). Myosin V walks hand-over-hand: single fluorophore imaging with 1.5-nm localization. Science 300, 2061-2065. doi: 10.1126/science.10 84398

Young, G., Hundt, N., Cole, D., Fineberg, A., Andrecka, J., Tyler, A., et al. (2018). Quantitative mass imaging of single biological macromolecules. Science 360, 423-427. doi: 10.1126/science.aar 5839
Young, G., and Kukura, P. (2019). Interferometric scattering microscopy. Annu. Rev. Phys. Chem. 70, 301-322. doi: 10.1146/annurev-physchem-050317-021247

Conflict of Interest: The authors declare that the research was conducted in the absence of any commercial or financial relationships that could be construed as a potential conflict of interest.

Copyright (๑ 2020 Taylor, Holler, Mahmoodabadi, Küppers, Dastjerdi, Zaburdaev, Schambony and Sandoghdar. This is an open-access article distributed under the terms of the Creative Commons Attribution License (CC BY). The use, distribution or reproduction in other forums is permitted, provided the original author(s) and the copyright owner(s) are credited and that the original publication in this journal is cited, in accordance with accepted academic practice. No use, distribution or reproduction is permitted which does not comply with these terms. 